\title{
MORTALIDAD EN LA FUERZA LABORAL COLOMBIANA DESDE EL ANÁLISIS EXPLORATORIO DE DATOS ESPACIALES
}

\author{
Mortality in colombian workforce from exploratory analysis of spatial data
}

\author{
Gabriel Alberto Agudelo Torres \\ Magíster en Matemáticas Aplicadas. Instituto Tecnológico Metropolitano. albertoagudelo@itm.edu.co
}

\section{Cómo citar /How to cite}

Agudelo Torres, G. A. (2015). Mortalidad en la fuerza laboral colombiana desde el análisis exploratorio de datos espaciales. Revista CEA, 1(1), 23-49.

Recibido: 22 de abril de 2014

Aceptado: 29 de julio de 2014

\section{Resumen}

Colombia perdió parte de su fuerza laboral por fallecimientos en el año 2011; las dos principales causas de esto son: los homicidios y sus secuelas, y las enfermedades isquémicas del corazón. Dichas causas de mortalidad presentan el fenómeno llamado dependencia espacial. Cuando el fenómeno de dependencia espacial está presente es posible utilizar técnicas como el análisis exploratorio de datos espaciales para determinar la localización geográfica que deben tener las políticas de seguridad y salud pública, en aras de disminuir la pérdida de capital humano por estos conceptos, maximizando, además, su efecto en las regiones vecinas.

En este artículo se cuantifica la interacción regional en el comportamiento de las tasas de defunción de personas en edad laboral. Se concluye que para el caso de los homicidios y sus secuelas, el norte de Antioquia y sur de Córdoba, Valle del Cauca y sus municipios vecinos, algunos otros del Cauca y Nariño, deben ser intervenidos con políticas integrales de seguridad. En cuanto a las enfermedades isquémicas del corazón, la intervención debe focalizarse en varias subregiones del país, teniendo presente que en el centro no solo debe atacarse este tipo de enfermedad sino también las enfermedades hipertensivas y las cerebrovasculares, pues estas tienen una alta influencia en las isquémicas del corazón.

Palabras clave: mortalidad, fuerza laboral, datos espaciales, homicidios, enfermedad isquémica del corazón.

\begin{abstract}
The two main reasons why Colombia lost part of its workforce due to deaths in 2011 are: murder and its aftermath and ischemic heart disease. These causes have the phenomenon called spatial dependence. When the phenomenon of spatial dependence is present it is possible to use techniques such as exploratory spatial data analysis to determine the geographical location which security and public health policies should have in order to reduce the loss of human capital for these reasons, while maximizing effect on neighboring regions.

This article quantifies regional interaction in the behavior of the death rates of people of working age. It is concluded that, in the case of the murders and their aftermath, northern Antioquia and southern Córdoba, Valle del Cauca and neighboring municipalities and some municipalities of Cauca and Nariño should be intervened with comprehensive security policies. As for ischemic heart disease, intervention should focus on several subregions of the country, bearing in mind that in the center of the country should be considered not only this type of disease, but also hypertensive and cerebrovascular diseases as these have a high influence in the ischemic heart disease.
\end{abstract}

Keywords: mortality, workforce, spatial data, homicide, ischemic heart disease. 


\section{INTRODUCCIÓN}

Para definir la fuerza laboral (o fuerza de trabajo) puede utilizarse el clásico Diccionario de Economía Política de Borísov, Zhamin y Makárova, el cual indica que es la capacidad de los habitantes para trabajar o el conjunto de fuerzas físicas y espirituales de las cuales su población dispone y que utiliza en el proceso de producción de los bienes materiales (Borísov, et al., 1966, p. 106). Una definición más práctica y acorde con nuestros tiempos puede ser: población económicamente activa, la cual se especifica formalmente como el conjunto de personas en edad de trabajar que trabajan o están buscando empleo.

La pérdida de esta fuerza laboral representa la reducción de la capacidad productiva total de un país, más, cuando se trata de personas con edades que llamaremos en adelante "edades laborales» y que para efecto de este artículo están entre los 15 y los 64 años de edad (si bien en Colombia la mayoría de edad se alcanza a los 18 años, la Ley 1098 de 2006 habilita a los mayores de 15 años para trabajar, siempre y cuando se cumplan algunas condiciones especiales tales como: contar con autorización por parte del Inspector de Trabajo o Ente Territorial Local, jornada diurna máxima de 6 horas diarias, entre otras). Dicha pérdida puede corresponder a mortalidad o invalidez en un grado considerable, producto de accidentes o enfermedades.

Para el caso colombiano, en el 2011 perdieron la vida 70.233 personas en edad laboral (personas entre los 15 y los 64 años), de estos, el $21,37 \%$ falleció por agresiones (homicidios) o por secuelas derivadas de estas, siendo la principal causa de mortalidad entre esa población. El 8,97\% falleció debido a enfermedades isquémicas del corazón, siendo la segunda causa de muerte y la primera en el caso de las enfermedades. El $69,66 \%$ restante falleció por diversas enfermedades 0 accidentes, que individualmente representan menos del $7 \%$ del total de muertes en esa población (cálculos propios).

Este artículo se centra entonces en las dos principales causas de pérdida de fuerza laboral en Colombia producto de fallecimientos: los homicidios y sus secuelas y las enfermedades isquémicas del corazón.

Uno de los objetivos de las políticas públicas es reducir la mortalidad de la población, lo cual incidiría positivamente en los indicadores de calidad de vida. En la medida en que se profundice en las causas de dicha mortalidad y en los efectos agregados que puede producir la implementación de programas especiales de atención en salud en zonas específicas del país, más eficiente será la asignación de recursos que los gobiernos destinan a estos rubros.

\section{MARCO TEÓRICO}

El análisis exploratorio de datos espaciales es una técnica aún poco utilizada entre los investigadores, al momento de poner en práctica la estadística descriptiva; esto a pesar del creciente interés por una rama de la econometría surgida en los años 40 llamada econometría espacial y cuyo desarrollo fue opacado hasta los años 80 por la econometría financiera, impulsada por Box y Jenkins a través de su obra Time series analysis. Forecasting and control (Agudelo, 2010, p. 5). Utilizar este tipo de econometría requiere demostrar en forma previa que la variable endógena contiene en sí misma un efecto denominado «dependencia espacial».

La dependencia espacial es el fenómeno que "surge siempre que el valor de una variable en un lugar del espacio está relacionado con su valor en otro u otros lugares del espacio» (Moreno y Vayá, 2000, p. 13). Detectar este fenómeno solo es posible si cada observación de la muestra o población tiene implícita la ubicación espacial de la misma, 
en cuyo caso dichas observaciones se denominan «datos espaciales».

En este contexto, el análisis espacial de las causas por las cuales Colombia pierde capital humano en su fuerza laboral, debido al fallecimiento del trabajador, cobra sentido pues factores económicos, alimenticios, culturales, entre otros, hacen que exista la posibilidad de dependencia espacial en las tasas de mortalidad de personas entre los 15 y los 64 años por cada 10.000 habitantes en las mismas condiciones. De ser validada dicha hipótesis, su uso sería necesario en el análisis exploratorio.

Los datos utilizados para el presente artículo corresponden al número de fallecidos en edad laboral (personas entre los 15 y los 64 años) durante el año 2011, por causa y municipio donde se registró dicho suceso, y a la proyección de población realizada por el DANE para ese año. Los primeros se extrajeron de los registros de los certificados individuales de defunción, consolidados en las bases de mortalidad del Departamento Administrativo Nacional de Estadística (DANE, 2012, p. 29).

Mediante la siguiente fórmula, se calculó la tasa de defunción de personas en edad laboral por cada 10.000 habitantes en la misma condición para cada municipio y causa de defunción. Dicha causa de defunción corresponde a diferentes grupos de enfermedades y sucesos agrupados, tal como se describe en la décima versión de la Clasificación Internacional de Enfermedades (CIE-10), publicada por la Organización Mundial de la Salud (World Health Organization, 1990).
Tasa de defunción $n_{M, c}=\frac{F_{M, c} * 10000}{\text { Población } n_{M}}$

Donde:

Tasa de defunción $n_{M, c}$ : Tasa de defunción de personas en edad laboral por cada 10.000 habitantes, en la misma condición para cada municipio $M$ y la causa de defunción $c$.

$F_{M, c}$ : Número de personas fallecidas en el municipio $M$ por la causa de defunción $c$.

Población $n_{M}$ : Población del municipio $M$.

Utilizando dichas tasas de defunción se detectaron comportamientos atípicos en algunos municipios del país, se calculó la interacción espacial entre esas unidades espaciales y se determinó si esta interacción produce altas o bajas tasas de mortalidad en regiones vecinas. Adicionalmente, para el caso de las enfermedades isquémicas del corazón, se analizó la relación espacial existente con otras enfermedades relacionadas.

\section{RESULTADOS Y DISCUSIÓN}

Partiendo de los cálculos descritos, se obtuvo la tasa total de defunción de personas en edad laboral por municipio, encontrando 11 de ellos en el percentil 99 (Ver Fig. 1). Dichos municipios se encuentran distantes el uno del otro, excepto los pertenecientes al Departamento del Valle del Cauca, donde Argelia, El Dovio, La Victoria y Caicedonia tienen fronteras en común (llamada contigüidad grado 1) o se interpone un solo municipio entre ellos (llamada contigüidad grado 2). Este hecho puede ser indicativo de la presencia de dependencia espacial entre dichos municipios. 


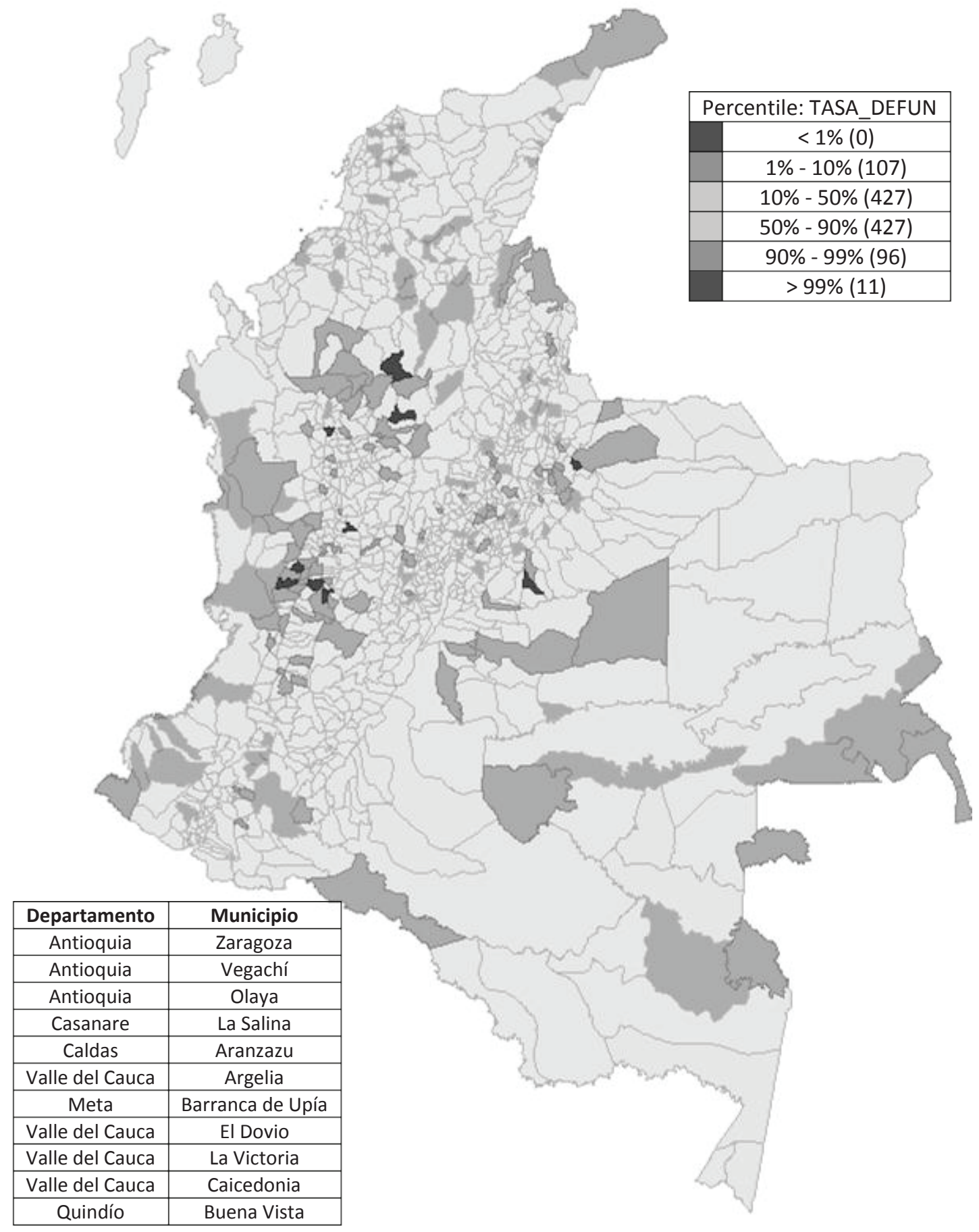

Figura 1. Distribución por municipios de la tasa de defunción de personas entre los 15 y los 64 años por cada 10.000 habitantes en la misma condición agrupados por percentiles. En la tabla, municipios pertenecientes al percentil 99 según la definición usual de cuantil (Wackerly, Mendenhal y Scheaffer, 2010, p. 164).

Figure 1. Distribution by municipalities of the rate of death for people between 15 and 64 per 10,000 in the same condition grouped by percentiles. In the table, municipalities in the 99th percentile according to the usual definition of quantile (Wackerly, and Scheaffer Mendenhal, 2010, p. 164). 
Tal como lo indica la teoría del análisis exploratorio, el hecho de tener una observación en un cuantil determinado no es indicativo de la presencia de datos atípicos o datos extremos dentro de la muestra. De existir dichos datos en muestras de carácter espacial, es posible individualizarlos utilizando los cálculos propuestos por Hendra (Hendra y Staum, 2010, p. 3). Dichos cálculos se describen a continuación:

$$
\begin{aligned}
& \text { Dato atípico superior } \geq Q_{3}+1.5 *\left(Q_{3}-Q_{1}\right) \\
& \text { Dato atípico inf erior } \leq Q_{1}-1.5 *\left(Q_{3}-Q_{1}\right) \\
& \text { Dato extremo superior } \geq Q_{3}+3 *\left(Q_{3}-Q_{1}\right) \\
& \text { Dato extremo inferior } \leq Q_{1}-3 *\left(Q_{3}-Q_{1}\right)
\end{aligned}
$$

Donde $Q_{1}$ y $Q_{3}$ representan los cuartiles 1 y 3 de la muestra respectivamente.

Como se observa en la Fig. 2, el municipio de Abriaquí (Antioquia) si bien no pertenece al percentil 99, es un dato atípico superior a nivel país, pues su tasa de mortalidad en personas en edad laboral supera el umbral a partir del cual puede ser catalogado de esa forma. Los demás municipios identificados como atípicos superiores corresponden a los mismos ubicados en el percentil 99. Datos atípicos inferiores no se encontraron en la muestra

En cuanto a datos extremos (Ver Fig. 3), La Salina (Casanare) posee un nivel de mortalidad extremadamente alto comparado con la distribución del resto de las observaciones, llegando a niveles de 95,01 personas entre los 15 y los 65 años fallecidas por cada 10.000 habitantes en iguales condiciones. Este hecho es particularmente preocupante, pues los dos municipios que le siguen se ubican en un rango entre tasas del 68,4 y 72,2 (Zaragoza en Antioquia y Barranca de Upía en Meta).

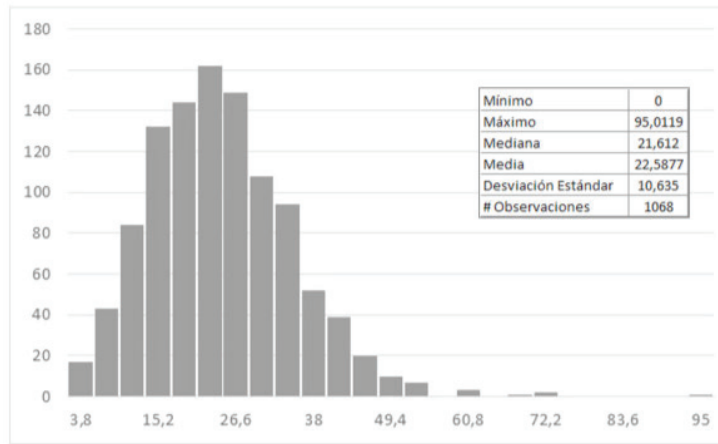

Figura 4. Distribución de frecuencia de la tasa de defunción de personas entre los 15 y los 64 años por cada 10.000 habitantes en la misma condición.

Figure 4. Frequency distribution of the rate of death for people between 15 and 64 per 10,000 population in the same condition.

Al tener esta distribución una cola larga a la derecha, como se observa en la Fig. 4, es de esperarse que los municipios que confirman dicha cola se desvíen mucho de la media de 22,59 fallecidos por cada 10.000 habitantes. 


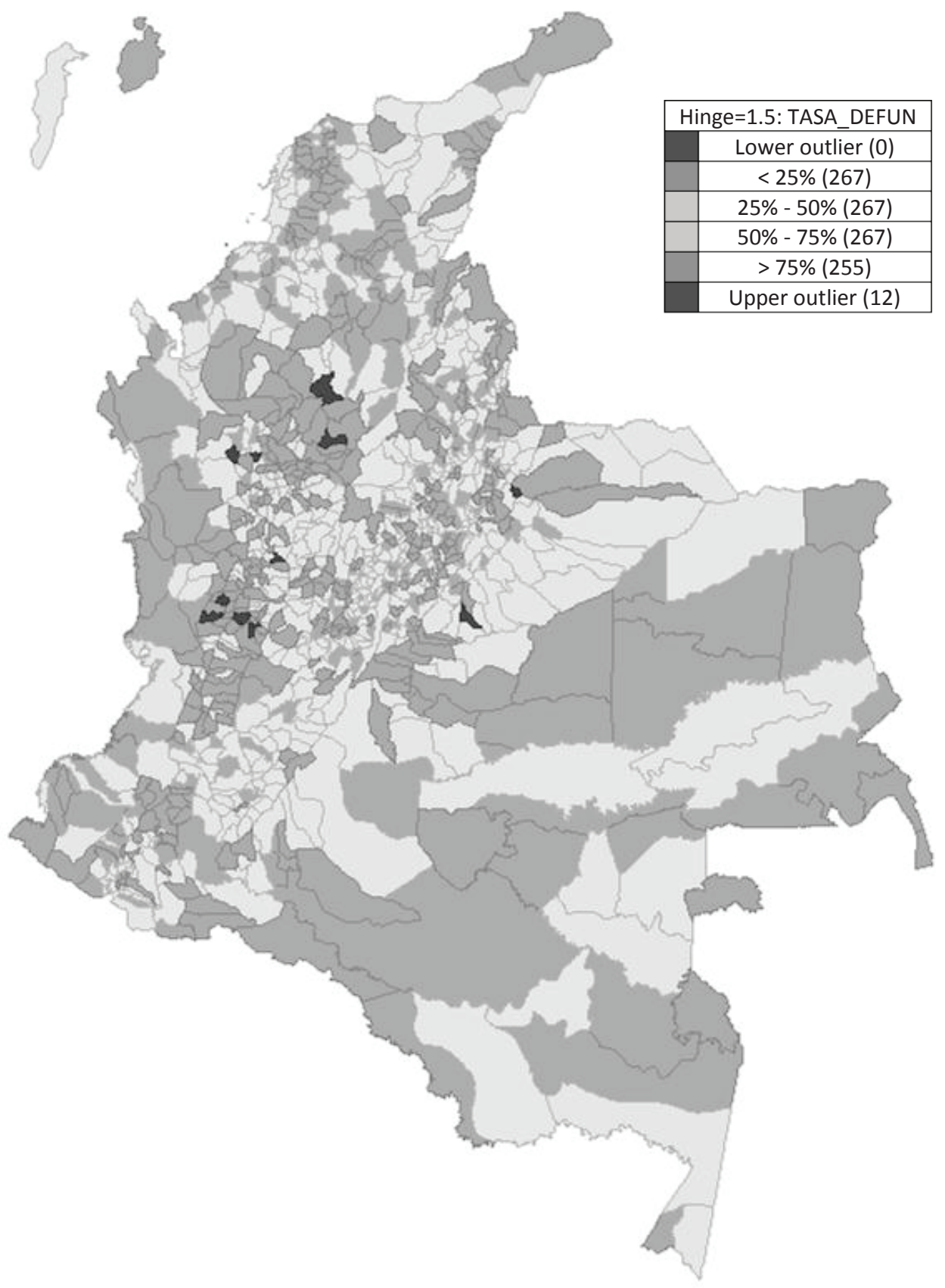

Figura 2. Distribución por municipios de la tasa de defunción de personas entre los 15 y los 64 años por cada 10.000 habitantes en la misma condición agrupados por cuartiles y clasificados como datos atípicos o no atípicos. En la tabla adjunta, municipios catalogados como datos atípicos superiores según cálculos propuestos por Hendra (Hendra y Staum, 2010, p. 3).

Figure 2. Distribution by municipalities of the rate of death for people between 15 and 64 per 10,000 in the same condition grouped by quartiles and classified as outliers or not outliers. In the table below, municipalities classified as upper outliers according to calculation proposed by Hendra (Hendra and Staum, 2010, p. 3). 


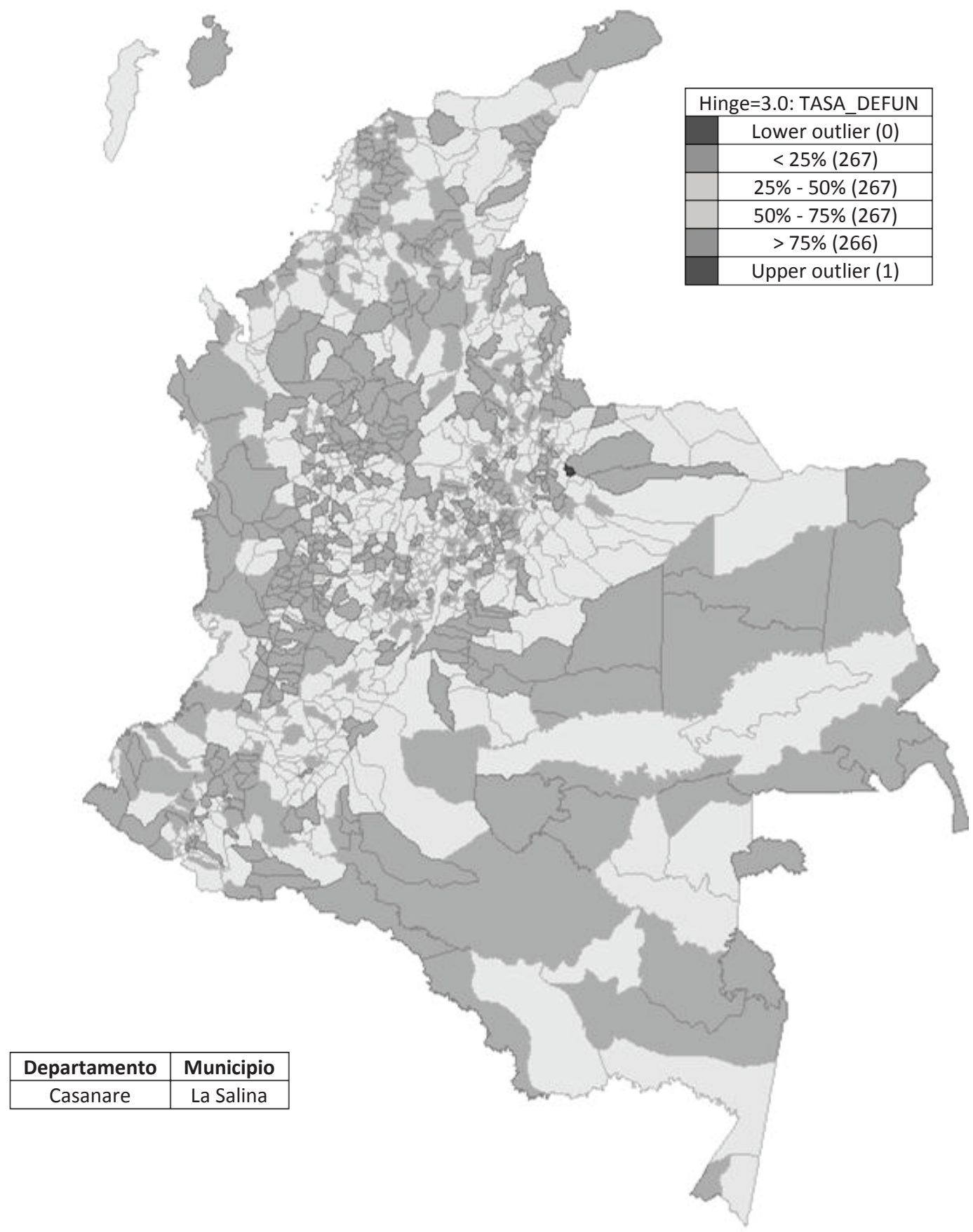

Figura 3. Distribución por municipios de la tasa de defunción de personas entre los 15 y los 64 años por cada 10000 habitantes en la misma condición agrupados por cuartiles y clasificados como datos extremos o no extremos. En la tabla adjunta, municipio catalogado como dato extremo superior según cálculos propuestos por Hendra (Hendra y Staum, 2010, p. 3).

Figure 3. Distribution by municipalities of the rate of death for people between 15 and 64 per 10,000 population in the same condition grouped by quartiles and classified as extreme or not extreme. In the table below, municipality listed as upper extreme data according to calculation proposed by Hendra (Hendra and Staum, 2010, p. 3). 


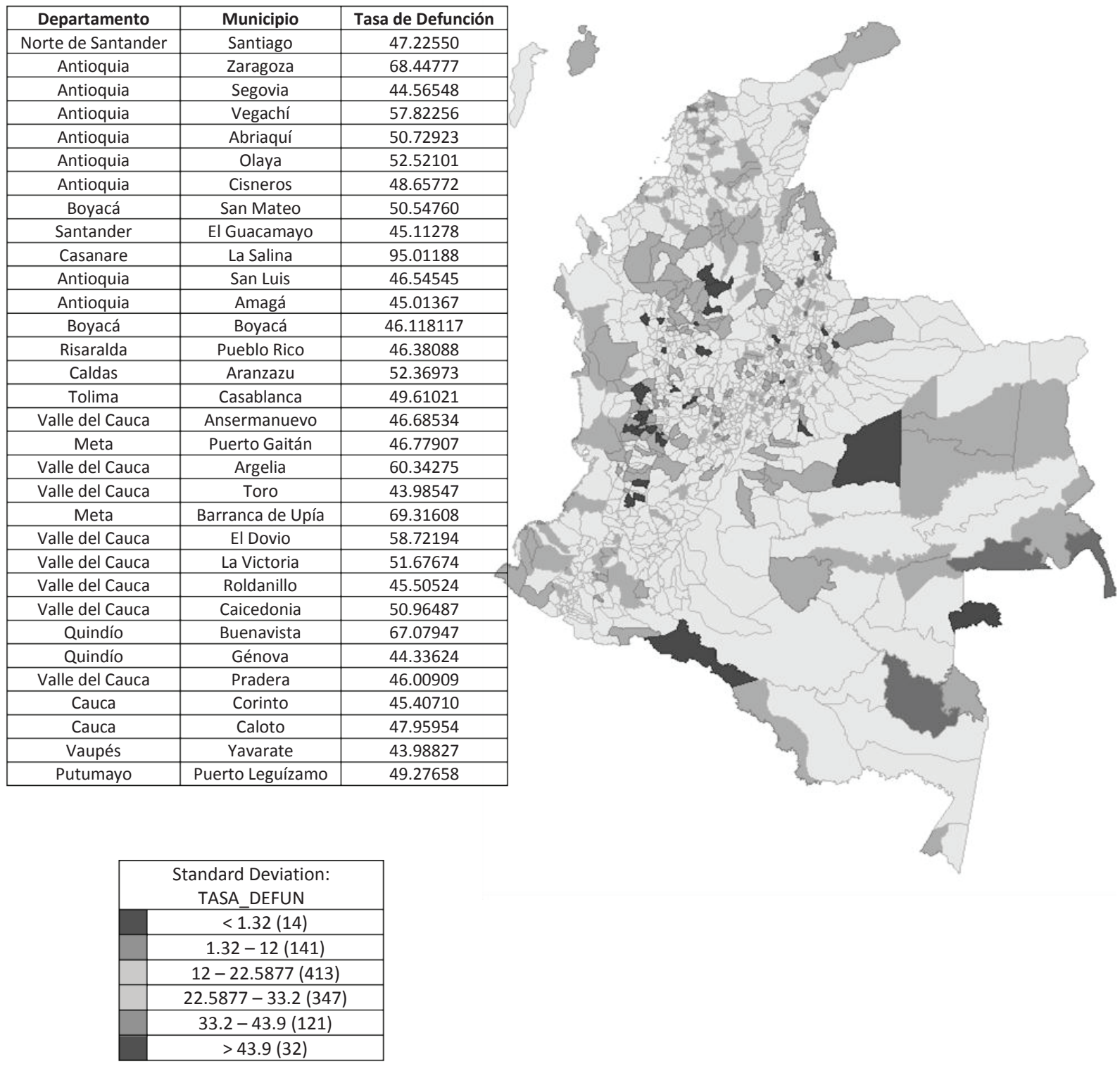

Figura 5. Distribución por municipios de la tasa de defunción de personas entre los 15 y los 64 años por cada 10.000 habitantes en la misma condición, agrupados por cantidad de desviaciones estándar. En la tabla, municipios que se desvían 43,9 fallecidos en promedio con respecto a la media de 22,59 que se muestra en la Figura 4.

Figure 5. Distribution by municipalities of the rate of death for people between 15 and 64 per 10,000 population in the same condition, grouped by number of standard deviations. In the table, municipalities whose deviation on average is 43.9 deceased with respect to the mean of 22.59 as is shown in Figure 4. 
Como resultado, se observan 32 municipios que aportan cada uno en promedio 43,9 personas fallecidas por cada 10.000, habitantes por encima de la media nacional de 22,59. La Fig. 5 indica que dichos municipios se ubican principalmente en los departamentos de Antioquia y Valle del Cauca, siendo los del segundo departamento, vecinos en primer o segundo grado de contigüidad con Pueblo Rico (Risaralda), Buenavista (Quindío) y Génova (Quindío), conformando así una subregión de alta siniestralidad que se detalla en la Fig. 6 y cuyas causas de existencia se explicarán más adelante.

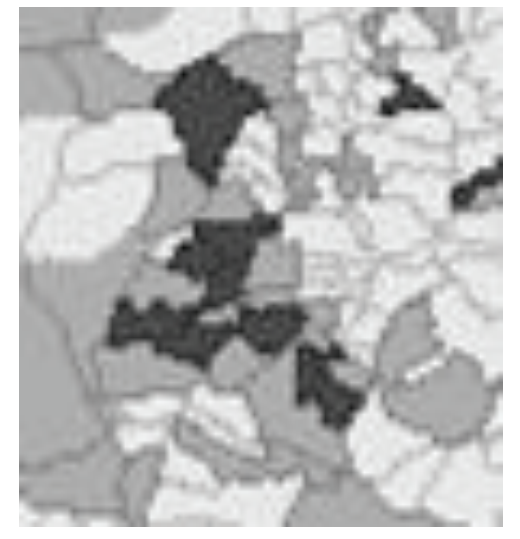

\begin{tabular}{|c|c|}
\hline Departamento & Municipio \\
\hline Risaralda & Pueblo Rico \\
\hline Valle del Cauca & Ansermanuevo \\
\hline Valle del Cauca & Argelia \\
\hline Valle del Cauca & Toro \\
\hline Valle del Cauca & El Dovio \\
\hline Valle del Cauca & La Victoria \\
\hline Valle del Cauca & Roldanillo \\
\hline Valle del Cauca & Caicedonia \\
\hline Quindío & Buenavista \\
\hline Quindío & Génova \\
\hline
\end{tabular}

Figura 6. Distribución por municipios del Valle del Cauca, Risaralda y Quindío de la tasa de defunción de personas entre los 15 y los 64 años por cada 10.000 habitantes en la misma condición, agrupados por cantidad de desviaciones estándar. En la tabla adjunta, detalle de los municipios que conforman dicha región.

Figure 6. Distribution by municipality of Valle del Cauca, Risaralda and Quindio of the rate of death for people between 15 and 64 per 10,000 population in the same condition, grouped by number of standard deviations. In the table below, details of the municipalities of the region.

Las causales de defunción que más afectan a estos 32 municipios son codificadas según la CIE-10, el homicidio y sus secuelas (causal 5.12) y las enfermedades isquémicas del corazón (causal 3.03). Estas causales coinciden perfectamente con las dos causales de fallecimiento que más inciden en la pérdida de fuerza laboral en Colombia y que reúnen el $30,4 \%$ del total de defunciones (homicidio y sus secuelas $21,4 \%$ y enfermedades isquémicas del corazón $9 \%$ ).

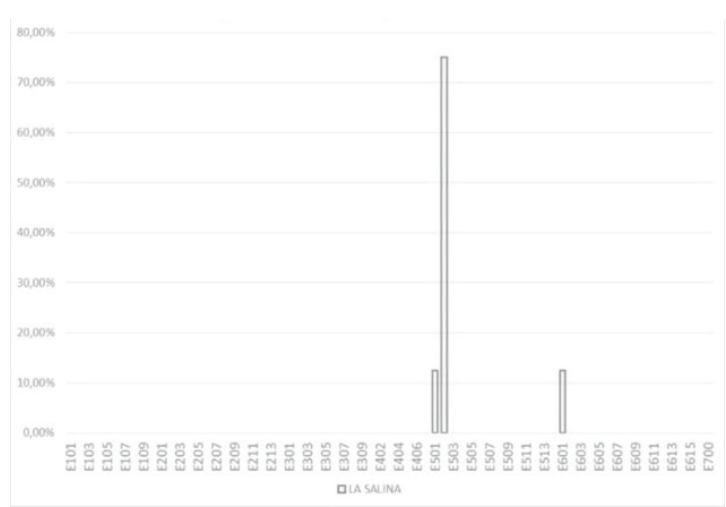

Figura 7. Porcentaje de fallecimientos de personas entre los 15 y los 64 años por cada 10000 habitantes en la misma condición, por causal de defunción en el municipio de La Salina (Casanare).

Figure 7. Percentage of deaths of people between 15 and 64 per 10,000 population in the same condition, for cause of death in the municipality La Salina (Casanare).

El análisis por causales de defunción por municipio (Ver Fig. 7) entrega un dato particular para el municipio de La Salina (Casanare) donde el $87,5 \%$ de los fallecimientos se producen por accidentes de transporte terrestre y por otros accidentes de transporte (causales 5.01 y 5.02). Si bien la tasa es alta, se debe tener presente que la escasa población entre 15 y 64 años de ese municipio (aproximadamente 842 personas) hace que los 6 fallecidos en accidentes de transporte terrestre y los 2 en otros 
accidentes de transporte constituyan ese porcentaje tan alto.

Analizar, entonces, las causas de mortalidad en la población colombiana entre 15 y 64 años debería comenzar por las dos causas que representan cerca de la tercera parte de los fallecimientos. Dichas causales se analizan a continuación.

\section{Causal 5.12 Agresiones homicidio, inclusive secuelas}

La distribución espacial de los homicidios en Colombia ha sido un tema ampliamente estudiado en nuestro país, pues es la dimensión natural en la que debe ser estudiada esta variable; sin embargo, aún quedan preguntas por resolver en cuanto a la dependencia espacial que se presenta en este tipo de fenómenos y cuáles deberían ser los focos de actuación para atacar este problema.
Inicialmente se pueden observar datos atípicos en ciertas regiones colombianas conformadas por municipios vecinos (Ver Fig. $8)$, lo cual supone algún grado de dependencia inicialmente se pueden observar datos atípicos en ciertas regiones colombianas conformadas por municipios vecinos (Ver Fig. 8), lo cual supone algún grado de dependencia espacial que debe ser probado. Probar dicha dependencia espacial implica la posibilidad de maximizar los recursos tendientes a reducir las tasas de homicidios en la fuerza laboral colombiana concentrándolos en ciertos municipios para proveer el máximo beneficio.

Los datos extremos (Ver Fig. 9) proporcionan una alerta a las autoridades sobre la necesidad de implementar planes de choque en dichos municipios para tratar de llevar a estas poblaciones a unos niveles considerados «normales» dentro del comportamiento nacional. 


\begin{tabular}{|c|c|}
\hline Departamento & Municipio \\
\hline Norte de Santander & Convención \\
\hline Córdoba & Ayapel \\
\hline Córdoba & Tierralta \\
\hline Córdoba & Montelíbano \\
\hline Antioquia & Cáceres \\
\hline Córdoba & Puerto Libertador \\
\hline Norte de Santander & Santiago \\
\hline Antioquia & Tarazá \\
\hline Antioquia & Zaragoza \\
\hline Antioquia & Segovia \\
\hline Antioquia & Anorí \\
\hline Antioquia & Valdivia \\
\hline Antioquia & Ituango \\
\hline Antioquia & Remedios \\
\hline Antioquia & Yarumal \\
\hline Antioquia & Briceño \\
\hline Antioquia & Campamento \\
\hline Antioquia & Uramita \\
\hline Antioquia & Murindó \\
\hline Antioquia & Vegachí \\
\hline Antioquia & Yolombó \\
\hline Antioquia & Yali \\
\hline Antioquia & Puerto Berrío \\
\hline Arauca & Tame \\
\hline Antioquia & Cisneros \\
\hline Antioquia & Heliconia \\
\hline Antioquia & Amagá \\
\hline Risaralda & Mistrató \\
\hline Risaralda & Pueblo Rico \\
\hline Tolima & Casablanca \\
\hline
\end{tabular}

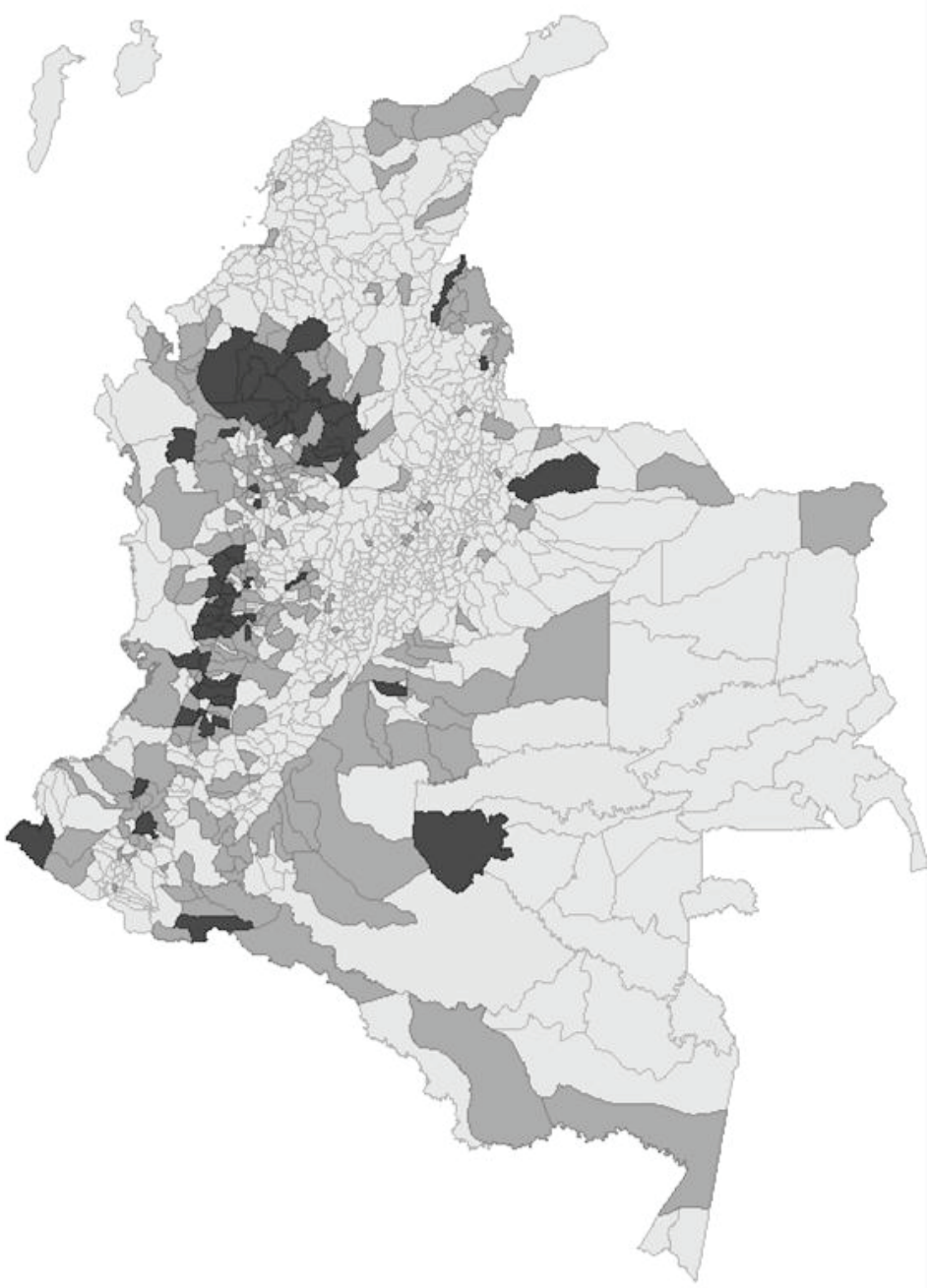

\begin{tabular}{|c|c|}
\hline Departamento & Municipio \\
\hline Chocó & San José del P. \\
\hline Caldas & Belalcázar \\
\hline Valle del Cauca & El Águila \\
\hline Valle del Cauca & Ansermanuevo \\
\hline Valle del Cauca & Argelia \\
\hline Valle del Cauca & Versalles \\
\hline Valle del Cauca & Toro \\
\hline Valle del Cauca & La Unión \\
\hline Quindío & Montenegro \\
\hline Valle del Cauca & El Dovio \\
\hline Valle del Cauca & La Victoria \\
\hline Valle del Cauca & Roldanillo \\
\hline Valle del Cauca & Zarzal \\
\hline Valle del Cauca & Bolívar \\
\hline Valle del Cauca & Caicedonia \\
\hline Valle del Cauca & Calima \\
\hline Valle del Cauca & Yotoco \\
\hline Valle del Cauca & El Cerrito \\
\hline Valle del Cauca & Palmira \\
\hline Meta & El Castillo \\
\hline Valle del Cauca & Yumbo \\
\hline Valle del Cauca & Pradera \\
\hline Valle del Cauca & Jamundí \\
\hline Cauca & Puerto Tejada \\
\hline Cauca & Corinto \\
\hline Cauca & Caloto \\
\hline Cauca & Argelia \\
\hline Guaviare & Calamar \\
\hline Cauca & Mercaderes \\
\hline Nariño & Colón \\
\hline Putumayo & Puerto Asís \\
\hline Nariño & Tumaco \\
\hline & \\
\hline & \\
\hline & \\
\hline & \\
\hline
\end{tabular}

\begin{tabular}{|c|c|}
\hline \multicolumn{1}{|c|}{ Hinge=1.5: E512 } \\
\hline & Lower outlier (0) \\
\hline & $<25 \%(0)$ \\
\hline $25 \%-50 \%(534)$ \\
\hline $50 \%-75 \%(267)$ \\
\hline$>75 \%(205)$ \\
\hline Upper outlier (62) \\
\hline
\end{tabular}

Figura 8. Distribución por municipios de la tasa de defunción por causal 5.12 de personas entre los 15 y los 64 años por cada 10.000 habitantes en la misma condición, agrupados por cuartiles y clasificados como datos atípicos o no atípicos. En la tabla adjunta, municipios catalogados como datos atípicos superiores según cálculos propuestos por Hendra (Hendra y Staum, 2010, p. 3).

Figure 8. Distribution by municipalities of the rate of death from causal 5.12 of people between 15 and 64 per 10,000 population in the same condition, grouped by quartiles and classified as outliers or not outliers. In the table below, municipalities classified as upper outliers according to calculation proposed by Hendra (Hendra and Staum, 2010, p. 3). 


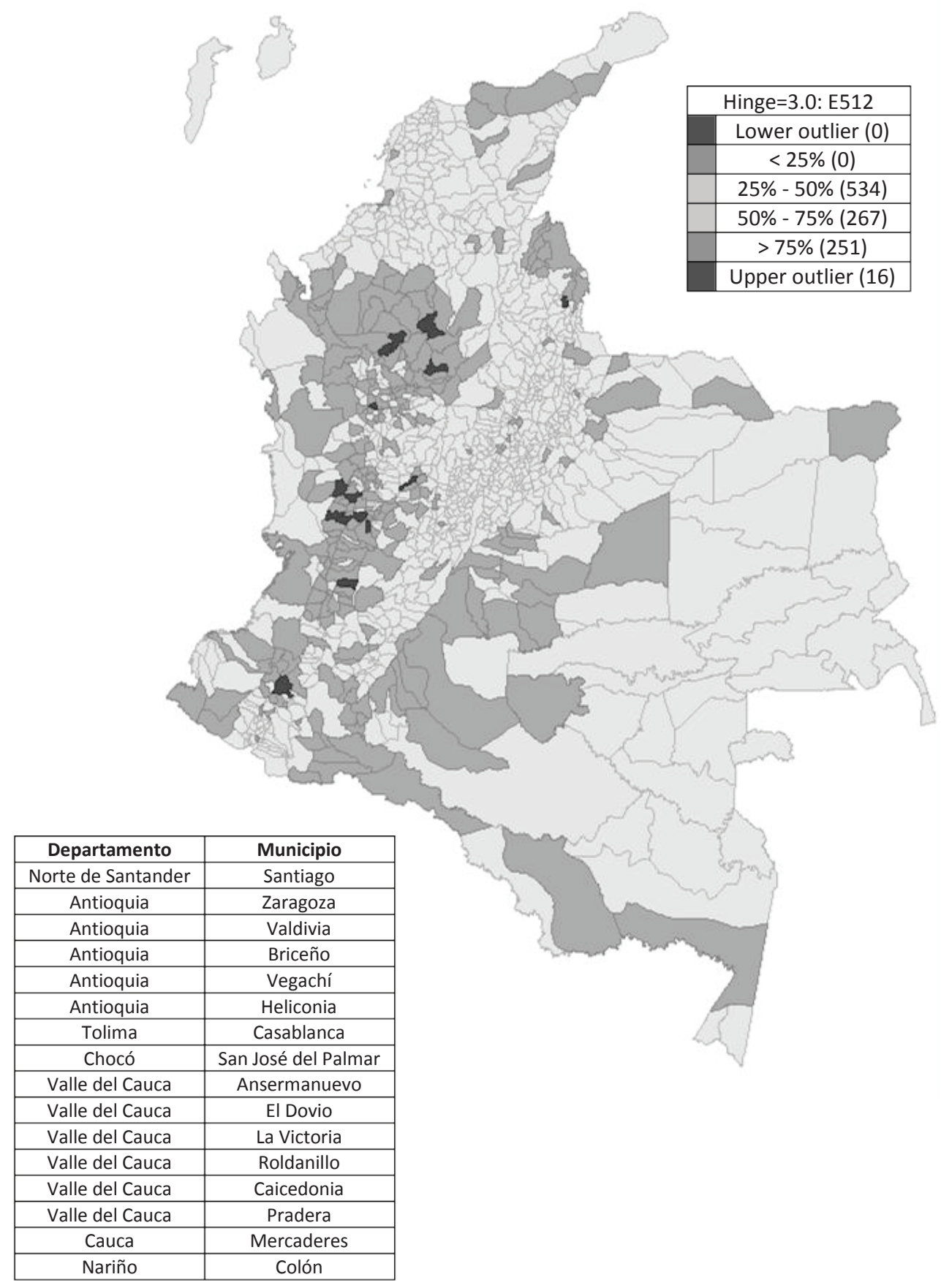

Figura 9. Distribución por municipios de la tasa de defunción por causal 5.12 de personas entre los 15 y los 64 años por cada 10.000 habitantes en la misma condición, agrupados por cuartiles y clasificados como datos extremos o no extremos. En la tabla adjunta, municipios catalogados como datos extremos superiores según cálculos propuestos por Hendra (Hendra y Staum, 2010, p. 3).

Figure 9. Distribution by municipalities of the rate of death from causal 5.12 of people between 15 and 64 per 10,000 population in the same condition, grouped by quartiles and classified as extreme or not extreme data. In the table below, municipalities classified as upper extreme data according to calculation proposed by Hendra (Hendra and Staum, 2010, p. 3). 
Para probar si existe algún tipo de dependencia espacial, usualmente se utiliza el test de Moran (Acevedo y Velásquez, 2008 , p. 22), el cual utiliza la siguiente prueba de hipótesis con su correspondiente estadístico. Sean:

$H_{0}$ : no existe dependencia espacial

$H_{1}$ : existe dependencia espacial

El estadístico de prueba es:

$$
Z=\frac{I-E[I]}{\sqrt{V[I]}} \sim \mathrm{N}(0,1)
$$

Donde $I=\frac{\sum_{i=1}^{N} \sum_{j=1}^{N} W_{i j}\left(y_{i}-\bar{y}\right)\left(y_{j}-\bar{y}\right)}{\sum_{i=1}^{N}\left(y_{i}-\bar{y}\right)^{2}}$, donde $\mathrm{N}(0,1)$ representa una distribución normal con media 0 y varianza $1, W_{i j}$ es la componente correspondiente a la fija $i$ y a la columna $j$ de la matriz de contigüidades estandarizada que se define más adelante, $N$ es el número de municipios de Colombia (1115), $E(I)=\frac{-1}{N-1}$ y $V[I]$ es la varianza de $I$.

Como es usual en la estadística espacial (tanto en técnicas de análisis exploratorio como en econometría espacial), el término $\mathrm{W}_{\mathrm{ij}}$ indica la componente ij de la matriz de contigüidades estandarizada (W). En dicha matriz, 0 representa la ausencia de contigüidad grado 1 entre las regiones $\mathrm{i}$ y $\mathrm{j}$ y un número $\delta$ el caso contrario, donde $\delta=\frac{1}{n}$ con $\mathrm{n}$ representando el número de vecinos de la región i.

Los resultados de este test (Ver Fig. 10) son concluyentes con un nivel de significancia $\alpha=0,001$ : se acepta la hipótesis alternativa correspondiente a la existencia de dependencia espacial en la tasas de homicidios municipales por cada 10.000 habitantes entre los 15 y los 64 años.

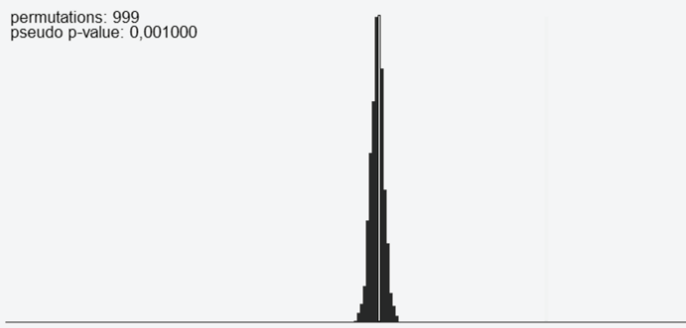

I: 0.4507 E[II: -0.0009 mean: -0.0011 sd: 0.0180 z-value: 25.1287

Figura 10. Distribución empírica de la tasa de defunción por causal 5.12 de personas entre los 15 y los 64 años por cada 10.000 habitantes en la misma condición y resultados del test de Moran definido por Agudelo (Agudelo, 2010, p. 23).

Figure 10. Empirical distribution of death rate per causal 5.12 of people between 15 and 64 per 10,000 population in the same condition and Moran's test results defined by Moran Agudelo (Agudelo, 2010, p. 23).

Este resultado corrobora que las condiciones socioeconómicas, geográficas, culturales, etc. tienen una fuerte influencia en los índices de violencia, representados en este caso por la tasa de homicidios en personas en edad laboral.

Sin embargo, es posible que algunas zonas del país no presenten dicha dependencia espacial, es decir, pueden existir algunas zonas "autónomas» donde los índices de violencia o seguridad no dependan del comportamiento de los municipios vecinos o algunas zonas donde la dependencia no sea tan acentuada como en otras. Para verificar este grado de dependencia espacial, comúnmente se utiliza la prueba de la I de Moran definida anteriormente como un indicador local de asociación espacial (LISA, por sus siglas en inglés). Dicho indicador identifica los clústeres de dependencia espacial existentes en un conjunto de unidades espaciales (en este caso municipios). 


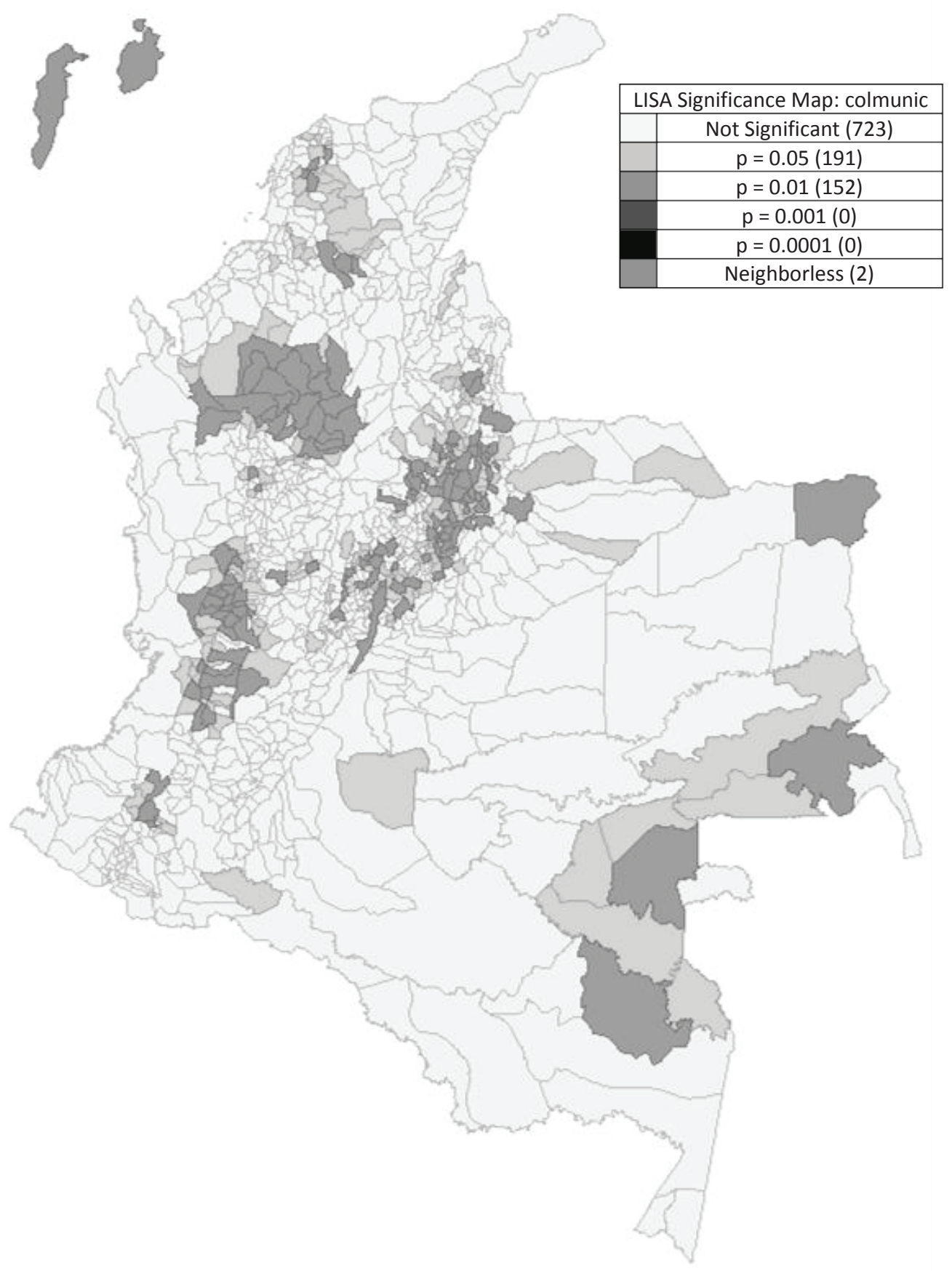

Figura 11. Distribución por municipios de la tasa de defunción por causal 5.12 de personas entre los 15 y los 64 años por cada 10.000 habitantes en la misma condición, agrupados por nivel de significancia del Test de Moran definido por Agudelo (Agudelo, 2010, p. 23).

Figure 11. Distribution by municipalities of the rate of death from causal 5.12 of people between 15 and 64 per 10,000 population in the same condition, grouped by level of significance of the Moran's test defined by Agudelo (Agudelo, 2010, p. 23). 
El análisis local detallado en la Fig. 11 indica una fuerte dependencia espacial (probabilidad de existencia del 0.99) en el norte de Antioquia y en la zona sur de Córdoba; en el Valle del Cauca y algunos municipios de Risaralda, Quindío, Caldas, Chocó, Tolima y Cauca; en una amplia zona que abarca los departamentos de norte de Santander, Santander, Boyacá, Casanare y Cundinamarca y en algunas pequeñas zonas entre Atlántico, Magdalena y Bolívar, entre Amazonas y Vaupés y entre Cauca y Nariño. Contiguas a estas zonas, por lo general existen otras con dependencia espacial menos fuerte (probabilidad de existencia del $0,95)$ que se ven menos impactadas por la variabilidad en la tasa de homicidios de sus vecinas.

Es importante resaltar que la existencia de una marcada dependencia espacial no implica que dichos municipios tengan altos índices de homicidio, pues el análisis de dependencia se circunscribe solamente a determinar el grado de impacto que provoca la variabilidad de una variable seleccionada en las regiones contiguas. En este sentido puede presentarse lo que se denomina dependencia espacial positiva (valores altos o bajos de la variable entre municipios cercanos) o negativa (valores altos en una unidad espacial y bajos en las unidades cercanas, o viceversa

La Fig. 12 es concluyente al determinar que en las zonas correspondientes al norte de Antioquia y sur de Córdoba, Valle del Cauca y sus municipios vecinos y algunos municipios de Cauca y Nariño, la situación de violencia, medida como homicidios de fuerza laboral, es alta y tiene la capacidad de esparcirse por la región con una gran intensidad. Sin embargo, también debe pensarse en aprovechar dicha dependencia espacial para crear condiciones favorables que permitan disminuir el índice de violencia en algunos municipios de la subregión, las cuales rápidamente se traducirían en mayor bienestar para la subregión completa.

Por el contrario, los municipios con alta dependencia espacial en la zona caribe, en los departamentos de Norte de Santander, Santander, Boyacá, Casanare y Cundinamarca y entre Amazonas, Vaupés y Guainía, corresponde a regiones con bajas tasas de homicidio por cada 10.000 habitantes entre 15 y 64 años y cuya relativa paz se transfiere a zonas vecinas con una alta intensidad.

\section{Causal 3.03 - Enfermedades isquémicas del corazón}

La causal 3.03 en el año 2011 ocasionó en Colombia 6.299 fallecimientos de personas pertenecientes a la fuerza laboral del país. Esta causal agrupa eventos tales como anginas de pecho (que a su vez agrupa anginas inestables, anginas de pecho con espasmos documentados, otras formas de anginas de pecho y las anginas de pecho sin clasificar), infartos agudos de miocardio, infartos agudos de miocardio posterior, ciertas complicaciones del paciente seguidas de un infarto agudo de miocardio, otras enfermedades cardiacas isquémicas agudas (trombosis coronaria que no deriva en un infarto agudo de miocardio y síndrome de Dressler) y la enfermedad cardíaca isquémica crónica.

Siguiendo la misma metodología utilizada para analizar la causal 5.02, se detectaron los valores atípicos y los valores extremos en las tasas municipales de defunción por esta causa por cada 10.000 habitantes en edad laboral 


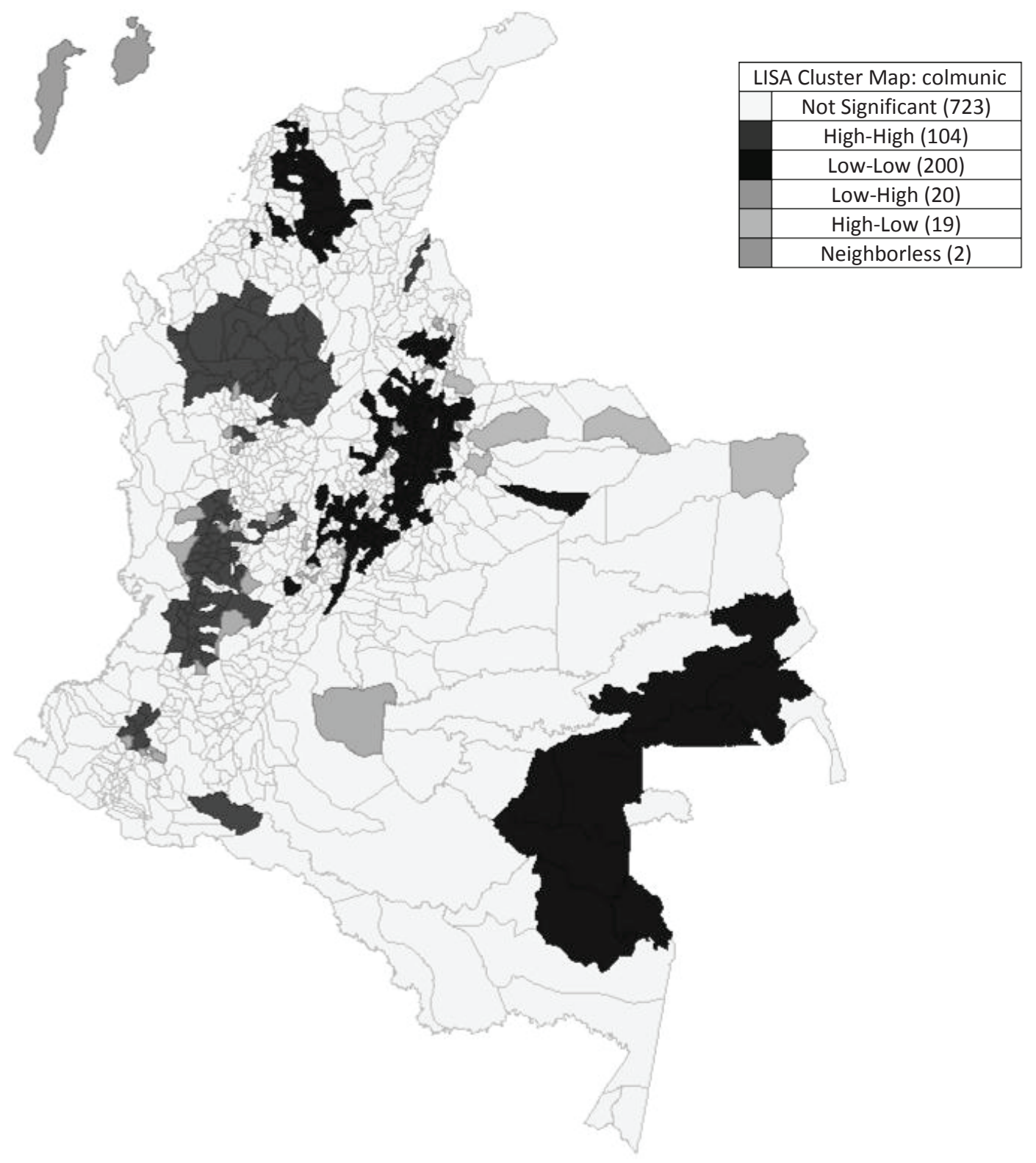

Figura 12. Distribución por municipios de la tasa de defunción por causal 5.12 de personas entre los 15 y los 64 años por cada 10.000 habitantes en la misma condición en municipios con dependencia espacial significativa (prob > 0.95), agrupados por clústeres según tasa del municipio y de sus vecinos.

Figure 12. Distribution by municipalities of the rate of death from causal 5.12 of people between 15 and 64 per 10,000 population in the same condition in municipalities with significant spatial dependence (prob>0.95), grouped by clusters according to municipality's rate and its neighbors's rate. 


\begin{tabular}{|c|c|}
\hline Departamento & Municipio \\
\hline Norte de Santander & Salazar \\
\hline Norte de Santander & Silos \\
\hline Antioquia & San José de la Montaña \\
\hline Santander & Molagavitas \\
\hline Boyacá & Guacamayas \\
\hline Boyacá & El Cocuy \\
\hline Antioquia & Armenia \\
\hline Boyacá & Sativasur \\
\hline Boyacá & San José de Pare \\
\hline Boyacá & Floresta \\
\hline Boyacá & Cuitiva \\
\hline Caldas & Marulanda \\
\hline Cundinamarca & Útica \\
\hline Boyacá & La Capilla \\
\hline Cundinamarca & Machetá \\
\hline Boyacá & Almeida \\
\hline Boyacá & Guayatá \\
\hline Cundinamarca & Gachetá \\
\hline Tolima & Murillo \\
\hline Meta & Barranca de Upía \\
\hline Tolima & Prado \\
\hline
\end{tabular}

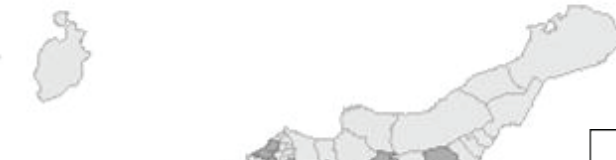

\begin{tabular}{|c|}
\hline Hinge $=1.5:$ E303 \\
\hline Lower outlier (0) \\
\hline$<25 \%(0)$ \\
\hline $25 \%-50 \%(534)$ \\
\hline $50 \%-75 \%(267)$ \\
\hline$>75 \%(246)$ \\
\hline Upper outlier (21) \\
\hline
\end{tabular}

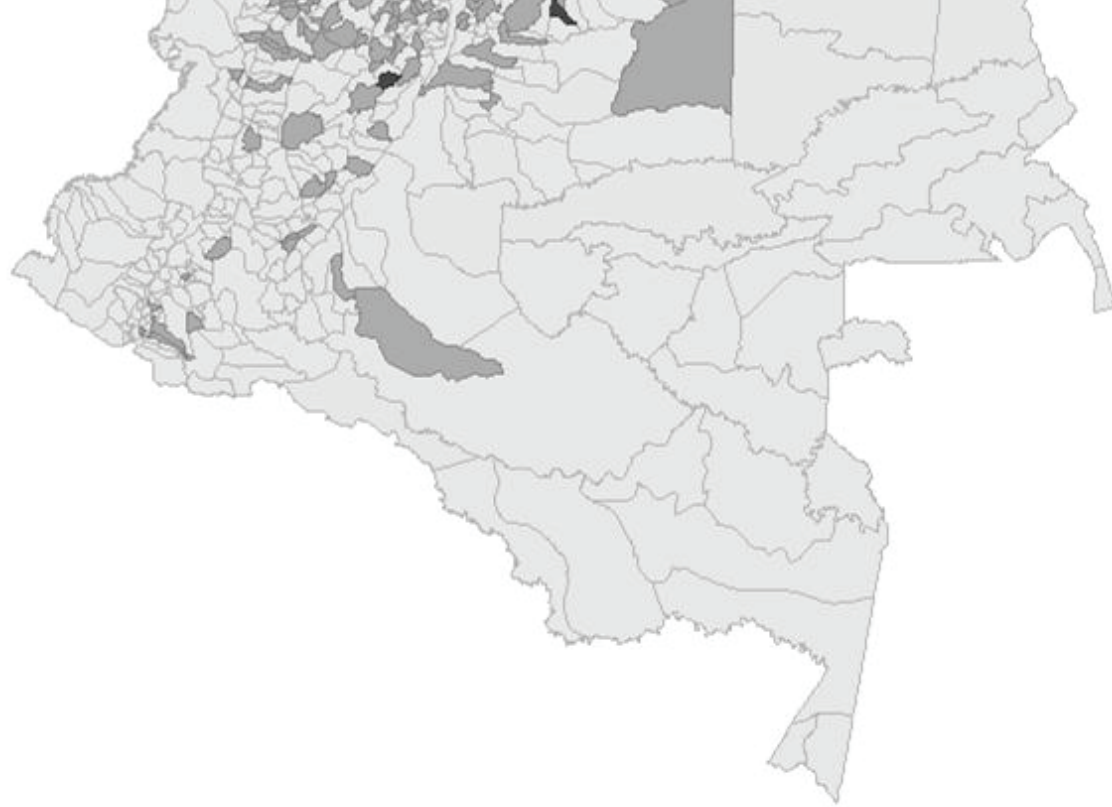

Figura 13. Distribución por municipios de la tasa de defunción por causal 3.03 de personas entre los 15 y los 64 años por cada 10.000 habitantes en la misma condición, agrupados por cuartiles y clasificados como datos atípicos o no atípicos según cálculos propuestos por Hendra (Hendra y Staum, 2010, p. 3).

Figure 13. Distribution by municipalities of the rate of death from causal 3.03 of people between 15 and 64 per 10,000 population in the same condition, grouped by quartiles and classified as outliers or not outliers according to calculation proposed by Hendra (Hendra and Staum, 2010, p. 3). 


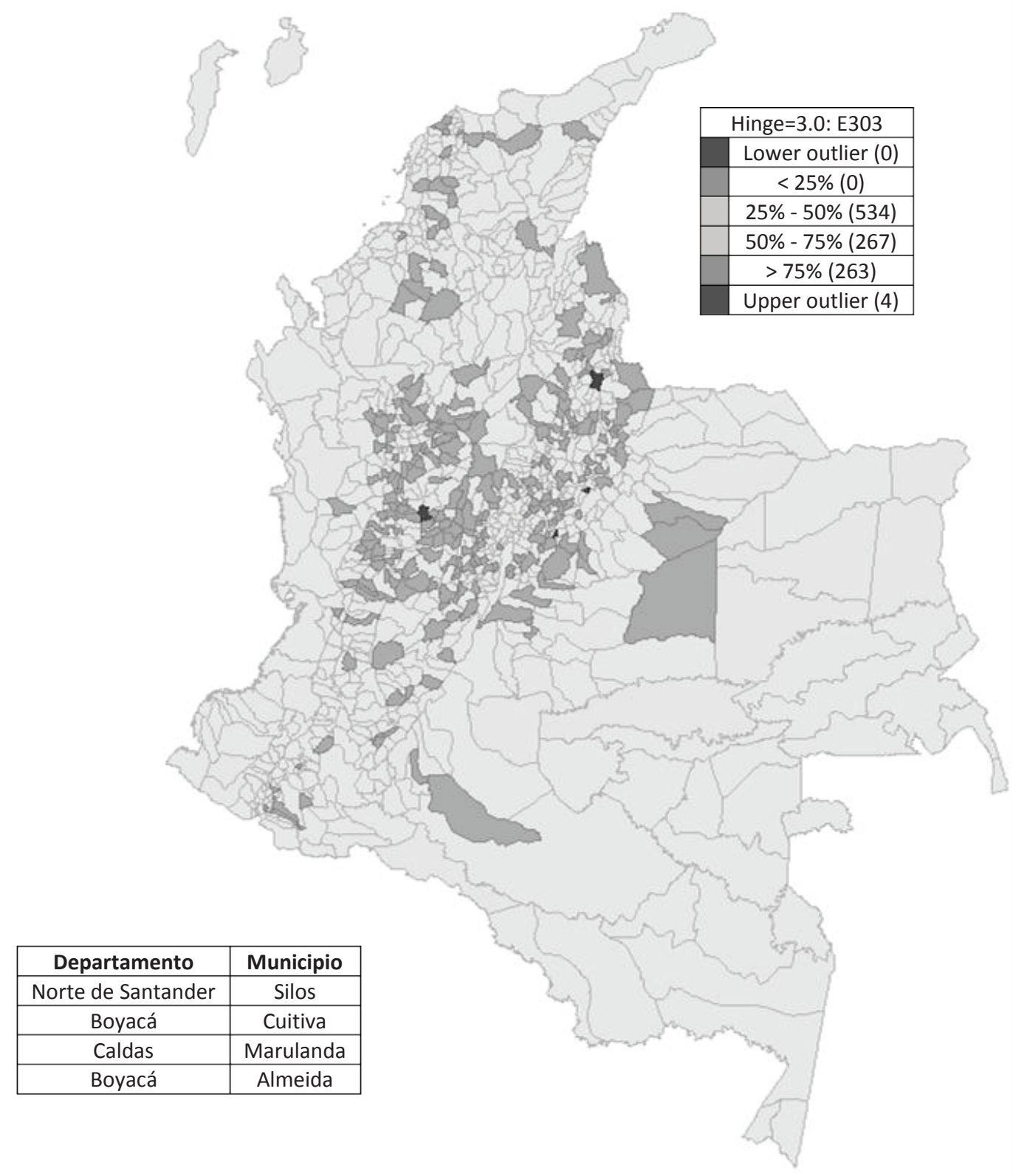

Figura 14. Distribución por municipios de la tasa de defunción por causal 3.03 de personas entre los 15 y los 64 años por cada 10.000 habitantes en la misma condición, agrupados por cuartiles y clasificados como datos extremos o no extremos según cálculos propuestos por Hendra (Hendra y Staum, 2010, p. 3).

Figure 14. Distribution by municipalities of the rate of death from causal 3.03 of people between 15 and 64 per 10,000 population in the same condition, grouped by quartiles and classified as extreme or not extreme data, according to calculation proposed by Hendra (Hendra and Staum, 2010, p. 3). 
Como se observa en las Fig. 13 y 14, los datos atípicos y los extremos no parecen estar localizados en una región en particular (salvo en una subregión compuesta por La Capilla, Almeida y Guayatá en Boyacá, y Machetá y Gachetá en Cundinamarca), por lo que sería fácil llegar a la conclusión errada sobre la inexistencia de dependencia espacial. Sin embargo, el test de la I de Moran, cuyos resultados se muestran en la Fig. 15, es claro al recomendar el rechazo de la hipótesis nula y la aceptación de la alternativa sobre la existencia de dependencia espacial.

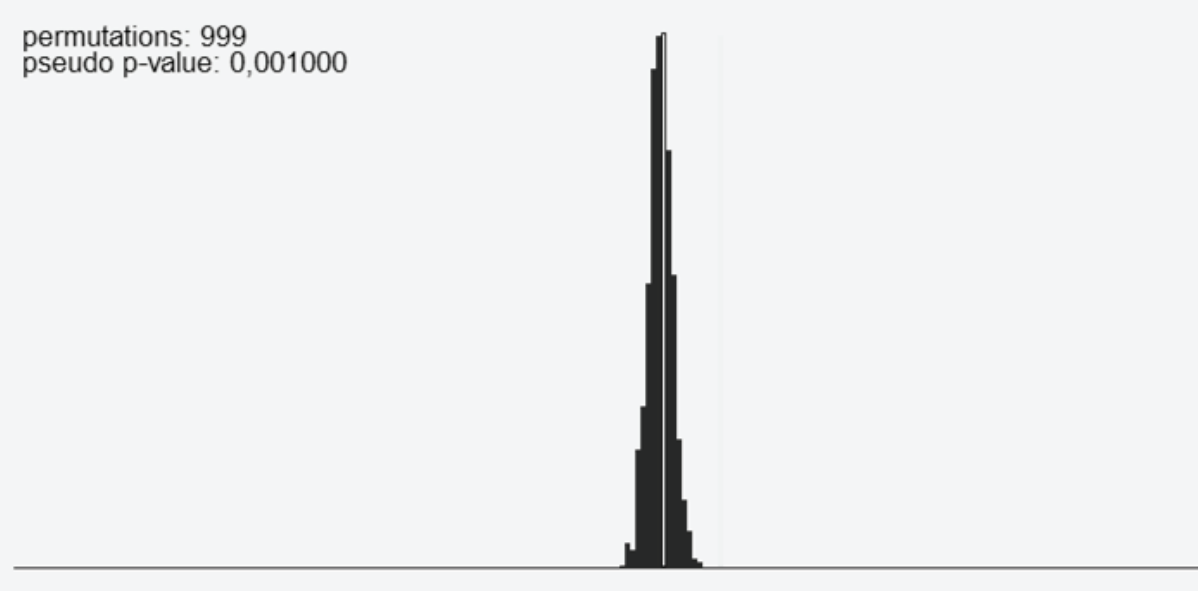

I: 0,0880 E[I]: $-0,0009$ mean: $-0,0010$ sd: 0,0184 z-value: 4,8296

Figura 15. Distribución empírica de la tasa de defunción por causal 3.03 de personas entre los 15 y los 64 años por cada 10.000 habitantes en la misma condición y resultados del test de Moran definido por Agudelo (Agudelo, 2010, p. 23).

Figure 15. Empirical distribution of death rate per causal 3.03 of people between 15 and 64 per 10,000 population in the same condition and Moran's test results defined by Agudelo (Agudelo, 2010, p. 23).

Desagregando por significancia de la prueba, se observan 38 municipios con una probabilidad de existencia de dependencia espacial del 0.99 y 151 con probabilidad de 0.95 (Ver Fig. 16). Estas regiones están concentradas en tres zonas básicamente: el sur del país, el departamento del Chocó y una amplia región del centro del país con tendencia hacia el eje cafetero.

El análisis de dependencia espacial positiva o negativa (Ver Fig. 17) entrega un resultado concluyente frente a la dependencia espacial positiva con valores altos en el centro occidente del país, a pesar de algunos municipios cercanos con dependencia espacial negativa. Este último fenómeno puede deberse a la migración de personas enfermas del corazón hacia municipios con mejores servicios de salud, lo cual debería provocar una disminución en las tasas del municipio origen y unas mayores tasas en el destino. 


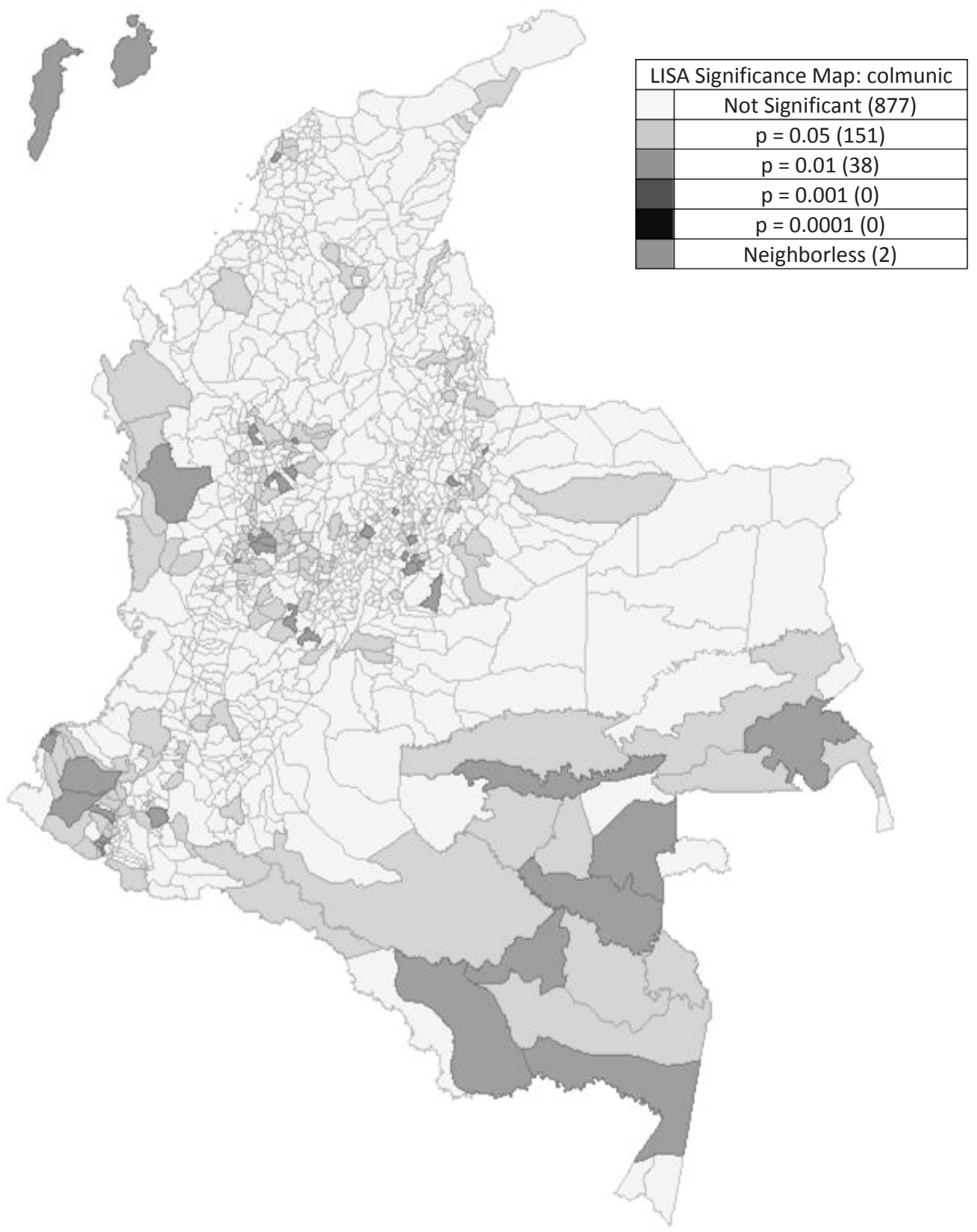

Figura 16. Distribución por municipios de la tasa de defunción por causal 3.03 de personas entre los 15 y los 64 años por cada 10.000 habitantes en la misma condición, agrupados por nivel de significancia del Test de Moran definido por Agudelo (Agudelo, 2010, p. 23).

Figure 16. Distribution by municipalities of the rate of death from causal 3.03 of people between 15 and 64 per 10,000 population in the same condition, grouped by level of significance of the Moran's test defined by Agudelo (Agudelo, 2010, p. 23). 


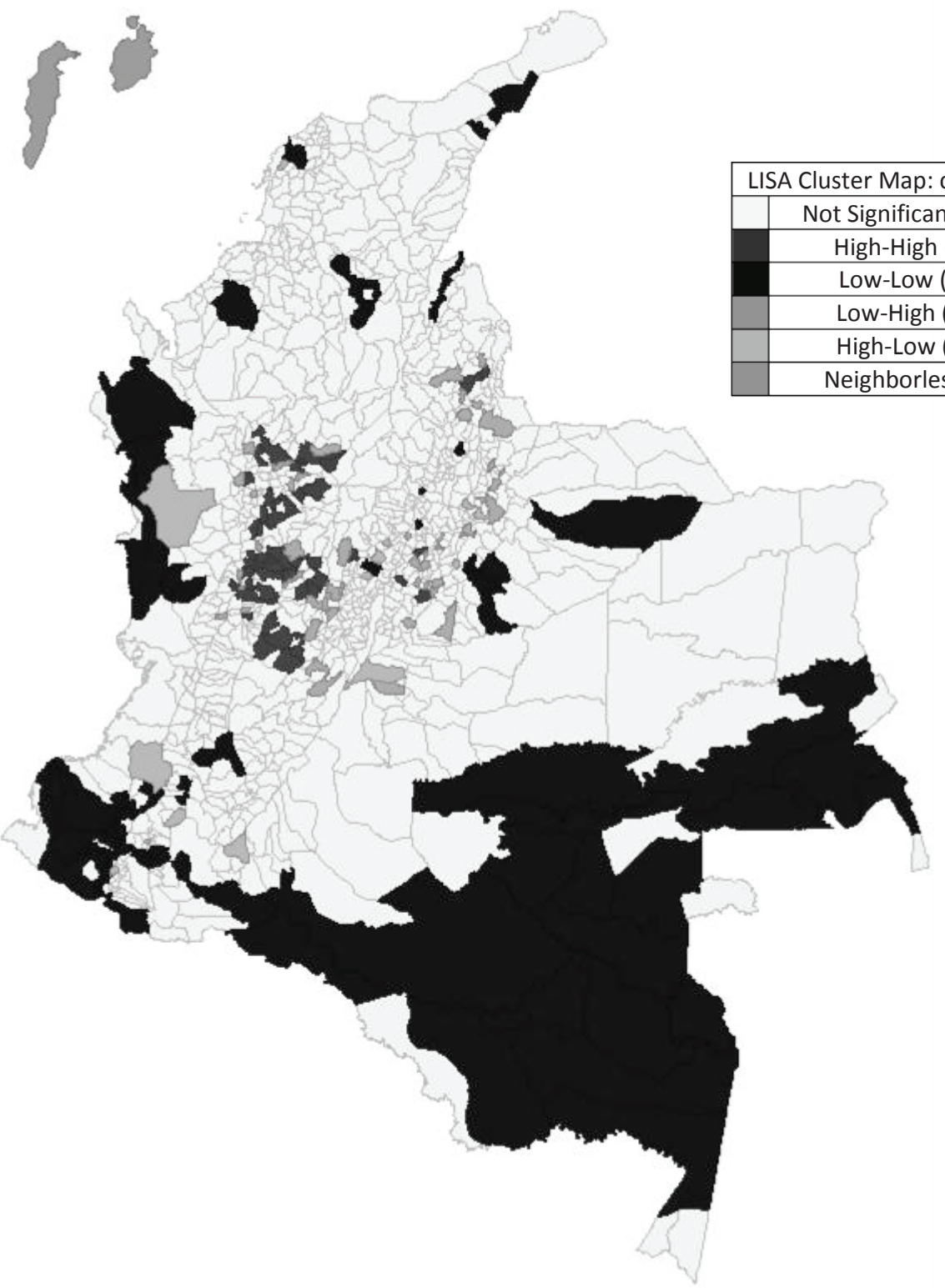

Figura 17. Distribución por municipios de la tasa de defunción por causal 3.03 de personas entre los 15 y los 64 años por cada 10.000 habitantes en la misma condición, en municipios con dependencia espacial significativa (prob > 0.95), agrupados por clústeres según tasa del municipio y de sus vecinos, según definición de vecindad tipo reina presentada por Acevedo (Acevedo y Velásquez, 2008, p. 18).

Figure 17. Distribution by municipalities of the rate of death from causal 3.03 of people between 15 and 64 per 10,000 population in the same condition, in municipalities with significant spatial dependence (prob>0.95), grouped by clusters according to municipality's rate and its neighbors's rate, using the queen neighborhood type defined by Acevedo (Acevedo and Velasquez, 2008, p. 18). 
El análisis univariado realizado, por su misma naturaleza, hace caso omiso a la posibilidad de una dependencia con otras variables que podrían ayudar a explicar la variabilidad en la variable endógena. Esto se debe tener en cuenta pues la causal 3.03 puede estar relacionada con otras causales de mortalidad cuyo origen sea hábitos comunes en la población, tales como mala alimentación, falta de ejercicio físico, entre otros.

Se contrastaron entonces dos causales de defunción que podrían tener incidencia en la variabilidad intermunicipal de la causal de defunción 3.03; dichas variables son la causal 3.02 -enfermedades hipertensivas- y la causa 3.07 -enfermedades cerebrovasculares-. Estas enfermedades deberían estar correlacionadas con la causal 3.03 enfermedades isquémicas del corazón- por cuanto las características sociodemográficas de las poblaciones influirían para que su población falleciera de enfermedades del sistema circulatorio.

Con el objetivo de corroborar dicha dependencia espacial, se utilizó un modelo mixto autorregresivo espacial de orden 1, arrojando resultados positivos para ambas variables bajo de hipótesis alternativa de dependencia espacial sustantiva (dependencia espacial causada por la omisión del retardo espacial en la variable endógena o en alguna de las variables exógenas ):

Test de dependencia espacial sustantiva:

Forma funcional:

$\operatorname{Causal}_{3.03}=\rho \operatorname{Waausal}_{3.03}+\operatorname{Causal}_{(3.02 \text { ó 3.07) }} \beta+\varepsilon$

Prueba de hipótesis:

$$
\begin{aligned}
& H_{0}: \rho=0 \\
& H_{a}: \rho \neq 0
\end{aligned}
$$

Estadístico:

$$
L M-L A G=\frac{\left(\frac{\varepsilon^{T} W \text { Causal }_{3.03}}{s^{2}}\right)^{2}}{n J} \sim \chi^{2}(1)
$$

Donde,

$n J=T+\frac{\left(\operatorname{WCausal}_{(3.02 \text { 6 } 3.07)} \beta\right)^{T} M\left(\operatorname{WCausal}_{(3.02 \text { o } 3.07)} \beta\right)}{S^{2}}$

$T=\operatorname{Tr}\left[\left(W+W^{T}\right) W\right]$

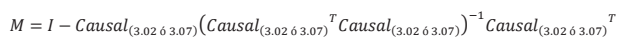

Causal $_{k}$ : Tasa de defunción debido a la causal $k$ de personas con edades entre los 15 y los 64 años por cada 10000 habitantes en la misma condición, con $k=3.02,3.03,3.07$.

$\rho$ : Coeficiente autorregresivo espacial (escalar), que recoge la intensidad de las interdependencias entre las observaciones muestrales.

$\beta$ : Escalar que recoge la intensidad de la relación entre la variable endógena y la variable exógena no autorregresiva.

$W$ : Matriz de contigüidades estandarizada.

$\varepsilon$ : Término de perturbación aleatoria.

$s^{2}$ : Varianza del término de perturbación aleatoria $\varepsilon$.

Resultado Dependencia espacial sustantiva Causal 3.02:

DIAGNOSTICS FOR SPATIAL DEPENDENCE

DIAGNOSTICS FOR SPATIAL DEPENDENCE
TPATIAI LAG DEPENDENCE FOR WEIGHT MATRIX : colmunic, gal
TEST

Resultado Dependencia espacial sustantiva Causal 3.07:

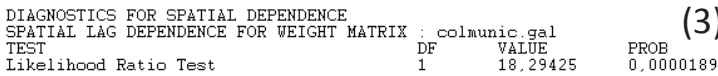

Al realizar un análisis bivariado local (BiLISA) a partir de la I de Moran se obtienen mapas de significancia de la dependencia espacial, incluyendo como variables explicativas de la variabilidad en la tasas de defunción por enfermedades isquémicas de corazón, la misma variable rezagada espacialmente y la causal 3.02 -enfermedades hipertensivas(Ver Fig. 18 y 19) y posteriormente la causa 3.07 -enfermedades cerebrovasculares- (Ver Fig. 20 y 21 ). 


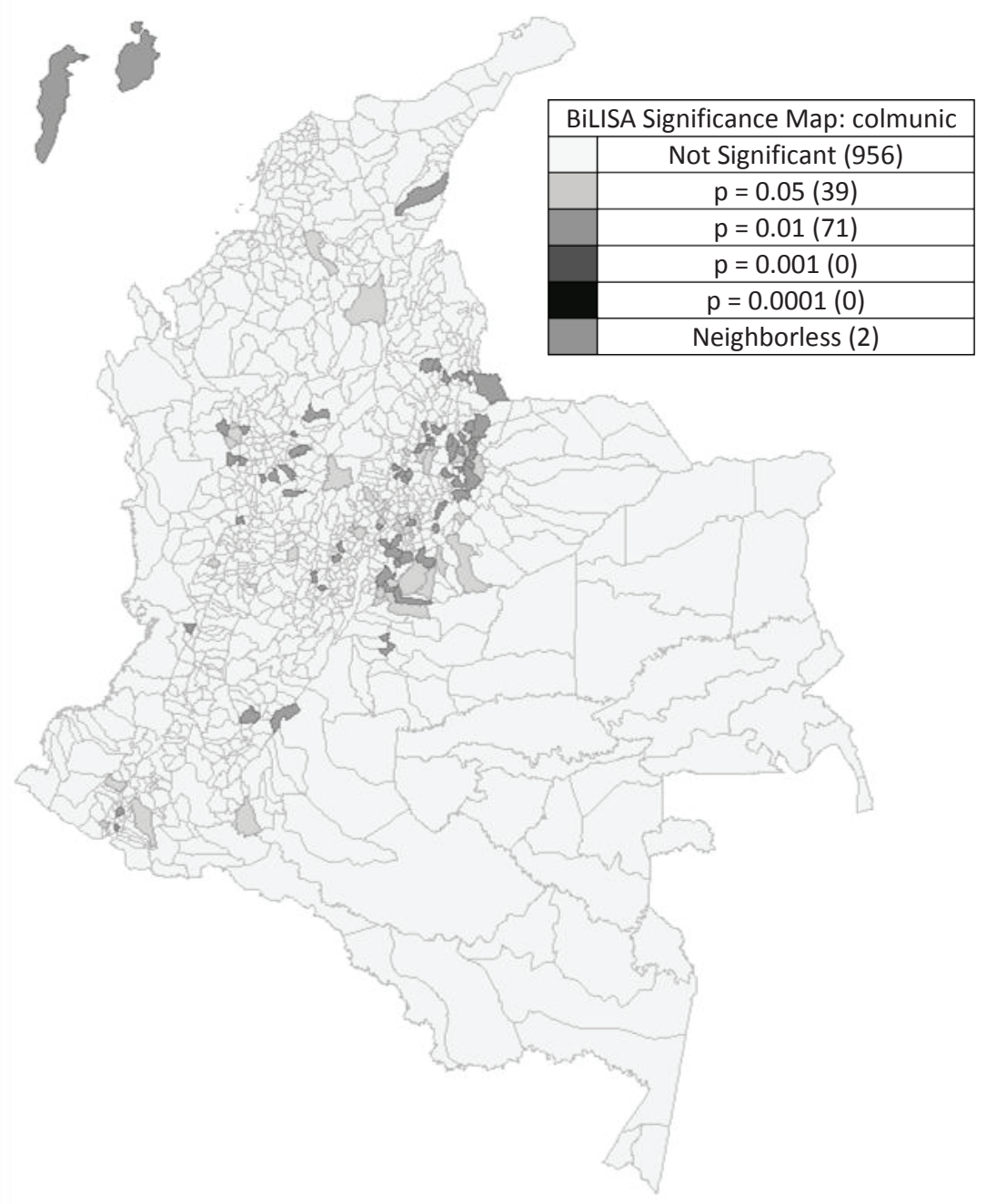

Figura 18. Distribución por municipios de la tasa de defunción por causal 3.03 de personas entre los 15 y los 64 años por cada 10.000 habitantes en la misma condición, agrupados por nivel de significancia del Test de Moran para dependencia espacial entre la variable endógena (causal 3.03), la variable endógena rezagada espacialmente y la causal 3.02.

Figure 18. Distribution by municipalities of the rate of death from causal 3.03 of people between 15 and 64 per 10,000 population in the same condition, grouped by level of significance of the Moran's test for spatial dependence between the endogenous variable (causal 3.03), the spatially lagged endogenous variable and the causal 3.02. 


\begin{tabular}{|c|c|}
\hline Departamento & Municipio \\
\hline Cesar & Pailitas \\
\hline Antioquia & Giraldo \\
\hline Antioquia & Antioquia \\
\hline Antioquia & Caicedo \\
\hline Santander & Valle de San José \\
\hline Santander & Palmas del Socorro \\
\hline Santander & Acamonte \\
\hline Antioquia & San Vicente \\
\hline Santander & Oiba \\
\hline Boyacá & Chita \\
\hline Boyacá & San Miguel de Sema \\
\hline Cundinamarca & Susa \\
\hline Cundinamarca & Guachetá \\
\hline Boyacá & Rondón \\
\hline Boyacá & Ventaquemada \\
\hline Boyacá & Ramiriquí \\
\hline Risaralda & Pueblo Rico \\
\hline Cundinamarca & La Peña \\
\hline Boyacá & Miraflores \\
\hline Boyacá & Pachavita \\
\hline Cundinamarca & Quebradanegra \\
\hline Tolima & Villa Hermosa \\
\hline Casanare & Sabanalarga \\
\hline Cundinamarca & Medina \\
\hline Cundinamarca & Fómeque \\
\hline Meta & Villavicencio \\
\hline Meta & \\
\hline
\end{tabular}

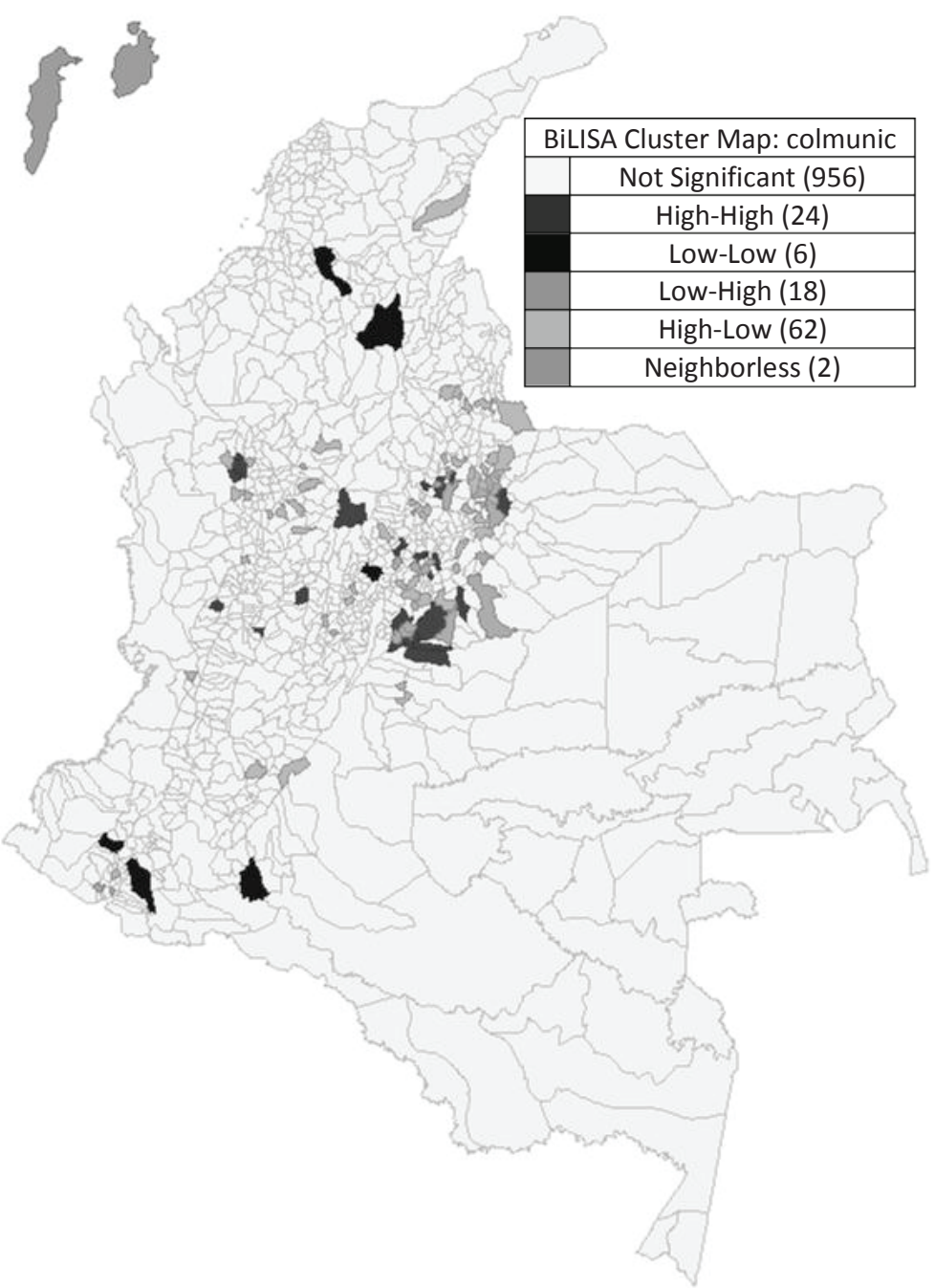

Figura 19. Distribución por municipios de la tasa de defunción por causal 3.03 de personas entre los 15 y los 64 años por cada 10.000 habitantes en la misma condición en municipios con dependencia espacial significativa (prob > 0.95) entre la variable endógena (causal 3.03), la variable endógena rezagada espacialmente y la causal 3.02, agrupados por clústeres según tasa del municipio y de sus vecinos, según definición de vecindad tipo reina presentada por Acevedo (Acevedo y Velásquez, 2008, p. 18).

Figure 19. Distribution by municipalities of the rate of death from causal 3.03 of people between 15 and 64 per 10,000 population in the same condition in municipalities with significant spatial dependence (prob> 0.95) between the endogenous variable (causal 3.03) the spatially lagged endogenous variable and the causal 3.02, grouped by clustes according to municipality's rate and its neighbors's rate, using queen neighborhood type defined by Acevedo (Acevedo and Velasquez, 2008, p. 18). 


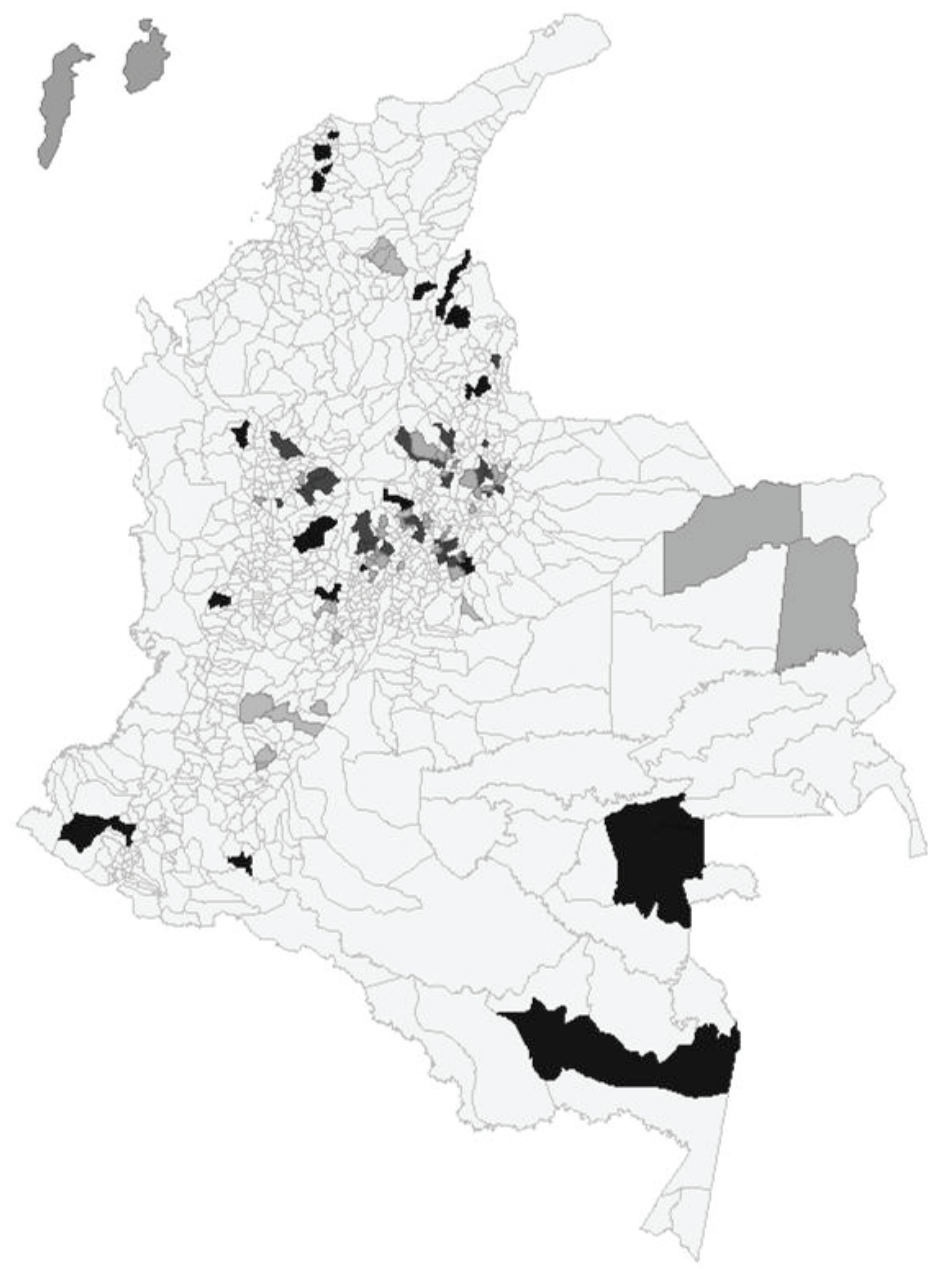

\begin{tabular}{|c|c|}
\hline \multicolumn{2}{|c|}{ BiLISA Cluster Map: colmunic } \\
\hline & Not Significant (964) \\
\hline & High-High (30) \\
\hline Low-Low (27) \\
\hline Low-High (30) \\
\hline High-Low (15) \\
\hline Neighborless (2) \\
\hline
\end{tabular}

\begin{tabular}{|c|c|}
\hline Departamento & Municipio \\
\hline Norte de Santander & San Cayetano \\
\hline Santander & Zapatoca \\
\hline Santander & Simacota \\
\hline Santander & Barichara \\
\hline Santander & Onzaga \\
\hline Antioquia & San Rafael \\
\hline Boyacá & Soata \\
\hline Santander & Oiba \\
\hline Antioquia & San Carlos \\
\hline Antioquia & Guatapé \\
\hline Antioquia & Granada \\
\hline Boyacá & Jericó \\
\hline Antioquia & Cocorná \\
\hline Boyacá & Sativasur \\
\hline Cundinamarca & Yacopí \\
\hline Boyacá & Saboya \\
\hline Boyacá & Sutamarchán \\
\hline Boyacá & San Miguel de Sema \\
\hline Boyacá & Ráquira \\
\hline Boyacá & Rondón \\
\hline Boyacá & Boyacá \\
\hline Cundinamarca & La Palma \\
\hline Cundinamarca & San Cayetano \\
\hline Cundinamarca & Topaipí \\
\hline Boyacá & Ramiriquí \\
\hline Boyacá & Ciénaga \\
\hline Boyacá & San Eduardo \\
\hline Cundinamarca & La Peña \\
\hline Boyacá & Chinavita \\
\hline Boyacá & Miraflores \\
\hline Tolima & Falán \\
\hline Valle del Cauca & Sevilla \\
\hline
\end{tabular}

Figura 21. Distribución por municipios de la tasa de defunción por causal 3.03 de personas entre los 15 y los 64 años por cada 10.000 habitantes en la misma condición, en municipios con dependencia espacial significativa (prob >0.95) entre la variable endógena (causal 3.03), la variable endógena rezagada espacialmente y la causal 3.07, agrupados por clústeres según tasa del municipio y de sus vecinos, según definición de vecindad tipo reina presentada por Acevedo (Acevedo y Velásquez, 2008, p. 18).

Figure 21. Distribution by municipalities of the rate of death from causal 3.03 of people between 15 and 64 per 10,000 population in the same condition, in municipalities with significant spatial dependence (prob> 0.95) between the endogenous variable (causal 3.03), the spatially lagged endogenous variable and the causal 3.07, grouped by clusters according to municipality's rate and its neighbors's rate, using queen neighborhood type defined by Acevedo (Acevedo and Velasquez, 2008, p. 18). 
La disminución de la tasa de fallecimientos en la fuerza laboral, por causa de enfermedades isquémicas de corazón, requiere de estrategias para atacar esta causa en los municipios correspondientes a la Fig. 17. Disminuirla en otros municipios con elevadas tasas de defunción por esta causal dependerá también de los esfuerzos realizados en atacar otras causas de enfermedades del sistema circulatorio, como las enfermedades hipertensivas y las causantes de decesos por enfermedades cerebrovasculares.

\section{CONCLUSIONES}

Casi la tercera parte de los fallecimientos en la fuerza laboral colombiana se debe a dos causas fundamentales: los homicidios y sus secuelas y las enfermedades isquémicas del corazón. Estos dos tipos de suceso causaron durante el año 201121.311 muertes en todo el territorio nacional, y sus repercusiones en términos de sociales y de pérdida de capital humano son incalculables.

El análisis de dependencia espacial aplicado a cada una de dichas causales de fallecimiento por municipio indica que hay una clara dependencia espacial; en el caso de los homicidios con ella misma y en caso de las enfermedades isquémicas del corazón con ella misma y con otro tipo de causales de mortalidad, tales como las enfermedades hipertensivas y las cerebrovasculares.

Para enfrentar las principales causas por las cuales Colombia pierde su fuerza laboral por fallecimiento, implica diseñar estrategias encaminadas a intervenir los municipios donde la existe una fuerte dependencia espacial, con tasas de mortalidad por dichas causas altas en el municipio candidato a ser intervenido y en sus vecinos, pues el efecto de dicha implementación de políticas tendrá el poder de esparcirse rápidamente por el espacio.
En el caso de los homicidios y sus secuelas, los municipios candidatos a ser intervenidos se ubican en el norte de Antioquia y sur de Córdoba, Valle del Cauca, sus municipios vecinos y algunos municipios de Cauca y Nariño. En cuanto a las enfermedades isquémicas del corazón, la intervención debe focalizarse en varias subregiones: en el sur del país, el departamento del Chocó y una amplia región del centro del país con tendencia hacia el eje cafetero; deben diseñarse políticas encaminadas a la prevención de enfermedades isquémicas del corazón en el centro del país, específicamente en los municipios detallados en las Fig. 19 y 21, no solo debe enfrentarse este tipo de enfermedad sino también las enfermedades hipertensivas y las cerebrovasculares, pues estas tienen una alta influencia en las isquémicas del corazón.

\section{REFERENCIAS}

Acevedo B., I., Velásquez C., E., (2008). Algunos conceptos de la econometría espacial y el análisis exploratorio de datos espaciales. Medellín: Universidad EAFIT.

Agudelo T., G.A. (2010). Dependencia espacial: detección, validación y modelación. Universidad EAFIT, Medellín.

Borísov, E. F., Zhamin, V. A., Makarova, M. F., Anísimov, G. D., Astájov, V. D., Atlas, M.S., Zlobin, I. D. (1966). Diccionario de economía política. Montevideo: Pueblos Unidos.

DANE - Departamento Administrativo Nacional de Estadística (2012). Metodología estadísticas vitales. Bogotá: Taller de Ediciones del DANE. 
Hendra, R. y Staum, P. (2010). A SAS Aplication to Identify and Evaluate Outliers. New York: Applications Development.

Instituto Nacional de Medicina Legal y Ciencias Forenses (2013, septiembre). Forensis 2012, datos para la vida. Bogotá: Quad Graphics.

Moreno S., R. y Vayá V., E. (2000). Técnicas econométricas para el tratamiento de datos espaciales: la econometría espacial. Barcelona: Edicions Universitat de Barcelona.
Wackerly, D., Mendenhall, W. y Scheaffer, R. (2010). Estadística matemática con aplicaciones. México: Cengage Learning Editores.

World Health Organization (1990, mayo). International Statistical Classification of Diseases and Related Health Problems 10th Revision (ICD-10). United Nations. Forty-third World Health Assembly, Genoa, Italy: United Nations. 